\title{
Contributions towards a conjecture of Erdős on perfect powers in arithmetic progression
}

\author{
N. Saradha and T. N. Shorey
}

\begin{abstract}
Let $n, d, k \geqslant 2, b, y$ and $\ell \geqslant 3$ be positive integers with the greatest prime factor of $b$ not exceeding $k$. It is proved that the equation $n(n+d) \cdots(n+(k-1) d)=b y^{\ell}$ has no solution if $d$ exceeds $d_{1}$, where $d_{1}$ equals 30 if $\ell=3 ; 950$ if $\ell=4 ; 5 \times 10^{4}$ if $\ell=5$ or $6 ; 10^{8}$ if $\ell=7$, 8,9 or $10 ; 10^{15}$ if $\ell \geqslant 11$. This confirms a conjecture of Erdös on the above equation for a large number of values of $d$.
\end{abstract}

\section{Introduction}

For an integer $\nu>1$, we define $P(\nu)$ to be the greatest prime factor of $\nu$ and we write $P(1)=1$. In this paper, we consider the equation

$$
\Delta=\Delta(n, d, k)=n(n+d) \cdots(n+(k-1) d)=b y^{\ell}
$$

in positive integers $n, d, k, b, y$ and $\ell$, where $d \geqslant 1, k \geqslant 2, \ell \geqslant 2, P(b) \leqslant k, \operatorname{gcd}(n, d)=1$ and $b$ is $\ell$-free. We observe that (1.1) has infinitely many solutions if $k=2$. Therefore, we always suppose that $k \geqslant 3$. Furthermore, Erdös and Selfridge [ES75] have completely solved (1.1) with $d=1$ for $P(b)<k$, Saradha [Sar97] has completely solved (1.1) for $P(b)=k$ with $k \geqslant 4$ and Győry [Győ98] has completely solved (1.1) for $P(b)=k$ with $k=3, \ell>2$. In the case $k=3, \ell=2$ the only solutions of (1.1) are given by $n=1,2,48$ as a consequence of some old Diophantine results, see [Sar98] for a history.

From now onwards we assume that $d>1$. Then we always suppose that $(n, d, k) \neq(2,7,3)$ so that $P(\Delta)>k$ by a result of Shorey and Tijdeman [ST90]. Erdös conjectured that (1.1) implies that $k$ is bounded by an absolute constant. Shorey [Sho00] showed that the above conjecture for $\ell \geqslant 4$ is a consequence of the $a b c$-conjecture. A stronger conjecture states the following.

Conjecture 1. Equation (1.1) implies that $(k, \ell)=(3,3),(4,2)$ or $(3,2)$.

On the other hand, it is known that (1.1) has infinitely many solutions if $(k, \ell)=(3,3),(4,2)$ or $(3,2)$, see Tijdeman [Tij89]. It was conjectured by Tijdeman that the number of triples $(n, d, k)$ satisfying (1.1) with $k>2, \ell>1, k+\ell>6$ is finite. Let $b=1$. Then Darmon and Granville [DG95] conjectured that $(1.1)$ implies that $(k, \ell)=(3,2)$, in which case we get parametric solutions given by $(n, d) \in\left\{\left(\left(t^{2}+2 t u-u^{2}\right)^{2}, 4 t u\left(u^{2}-t^{2}\right)\right),\left(2\left(t^{2}-u^{2}\right)^{2}, 6 t^{2} u^{2}-t^{4}-u^{4}\right)\right\}$ with $\operatorname{gcd}(t, u)=1$ and $t+u$ odd. The cases $(k, \ell)=(3,3),(4,2)$ are impossible by an old result of Euler, see [DG95]. When $d$ is fixed, Marszalek [Mar85] confirmed Erdős conjecture. When $\ell=2$, it has been proved that $d \geqslant 23$, $d \geqslant 31$ and $d \geqslant 105$ in Saradha [Sar98], Filakovszky and Hajdu [FH01] and Saradha and Shorey [SS03], respectively. From now onwards we assume that $\ell \geqslant 3$. Saradha [Sar97] showed that $d \geqslant 7$ unless $d=5, k=3$. Furthermore, Saradha and Shorey [SS01] showed that (1.1) with $k \geqslant 4$ does

Received 15 July 2003, accepted in final form 14 April 2004, published online 21 April 2005.

2000 Mathematics Subject Classification Primary 11D61.

Keywords: arithmetic progressions, Diophantine equations, elliptic equations.

This journal is (C) Foundation Compositio Mathematica 2005. 


\section{N. Saradha And T. N. Shorey}

not hold if $d$ is of the form $2^{a} 3^{b} 5^{c}$, where $a, b, c$ are non-negative integers. Let $d_{1}$ be given by

$$
d_{1}=\left\{\begin{array}{l}
30 \text { if } \ell=3 \\
950 \text { if } \ell=4 \\
5 \times 10^{4} \text { if } \ell=5,6 \\
10^{8} \text { if } \ell=7,8,9,10 \\
10^{15} \text { if } \ell \geqslant 11 .
\end{array}\right.
$$

We prove the following theorem.

Theorem 1. Assume (1.1) and $k \geqslant 4$ if $\ell>3$. Then $d>d_{1}$.

We have not been able to prove Theorem 1 for $k=3$ whenever $\ell>3$. From now onwards, we suppose that $k \geqslant 4$ whenever $\ell>3$. Theorem 1 confirms Conjecture 1 for a large number of values of $d$. For a survey of results on (1.1), we refer the reader to Shorey [Sho00, Sho02a, Sho02b]. We now give a plan of the proof of Theorem 1 . We assume (1.1) with $d \leqslant d_{1}$. Furthermore, by Lemma 1 , there is no loss of generality in assuming that the following hypothesis holds.

Hypothesis A. We have $d \leqslant d_{1}$ and either $k$ is prime or $k=4$ if $\ell>3$.

The proof depends on giving a good lower bound for

$$
\delta=\frac{n+(k-1) d}{k^{l+1}}
$$

say $\delta>\delta_{1}$. We achieve this by means of an iterative procedure in Lemma 3. On the other hand, we obtain an upper bound

$$
\delta<\delta_{2}
$$

by Lemmas 6 and 12. Furthermore, we compare the lower and upper bounds of $\delta$ in Lemma 13 to bound $\ell$ and $k$. Let $\ell$ and $k$ be fixed. By (1.3) and (1.4), we have

$$
\delta_{1} k^{\ell+1}<n+(k-1) d<\delta_{2} k^{\ell+1} .
$$

Let $\ell>3$. Then we use Algorithm 1 (see $\S 8$ ) to see that (1.1) is not satisfied for all values of $n$ given by (1.5). For $\ell=3$, we use Algorithm 2 ( $\operatorname{see} \S 10$ ). The iterative procedure referred above has its origin in [SS01, Lemma 3] and [SS03, Lemma 6]. Algorithm 2 is a refinement and an extension of the algorithm given in [SS01]. Algorithm 1 is a new contribution in this paper. Algorithms 1 and 2 provide a method for solving (1.1) whenever the variables $n, d, k, \ell$ are bounded. The bound $d_{1}$ we have given in Theorem 1 is not optimal. Increasing the value of $d_{1}$ would result in heavier computation. All of our computations are carried out with MATHEMATICA and we use SIMATH for solving certain elliptic equations in integers.

\section{Notation and preliminaries}

Let $q_{1}<q_{2}<\cdots$ be the sequence of all primes coprime to $d$ and let $p_{1}<p_{2}<\cdots$ be the sequence of all primes. We write $\pi_{d}(x)$ for the number of primes $\leqslant x$ and coprime to $d, \pi(x)$ for the number of primes $\leqslant x$. We use the estimates

$q_{i} \geqslant p_{i} \geqslant i \log i \quad$ for $i \geqslant 1 ; \quad \pi_{d}(x) \leqslant \pi(x) \leqslant \frac{x}{\log x}+\frac{1.5 x}{\log ^{2} x} \quad$ for $x \geqslant 1 ; \quad \pi(x)>\frac{x}{\log x} \quad$ for $x>17$.

See $\left[\right.$ RS62, p. 69] for the above inequalities. For an integer $x>0$, we write $q_{i}(x)=q_{\pi_{d}(x)+i}$ with $i \geqslant 1$. We set $\beta=\beta(d, k)=\prod_{p \mid d} p^{-\operatorname{ord}_{p}(k-1) !}$ and

$$
\beta_{1}=\beta_{1}(d, k)=(k-1) ! \beta .
$$




\section{PERFECT POWERS IN ARITHMETIC PROGRESSION}

For $s>0$ and $h \geqslant 0$, we define

$$
\begin{gathered}
\beta_{2}(s, h)=\beta_{2}(d, k, \ell, s, h)=k-\frac{(k-1) \log (k-1)+\log \beta}{\ell \log (k-1)+\log s-\log 2}-\pi_{d}(k)-h, \\
\beta_{3}(s, h)=\max \left(1,\left[\beta_{2}(s, h)\right]+1\right), \\
\beta_{4}(s, h)=\beta_{4}(d, k, \ell, s, h)=k-\frac{(k-1) \log (k-1)+\log \beta}{(\ell+1) \log (k-1)+\log s-\log 2}-\pi_{d}(k)-h
\end{gathered}
$$

and

$$
\beta_{5}(s, h)=\max \left(1,\left[\beta_{4}(s, h)\right]+1\right) .
$$

Since the left-hand side of (1.1) is divisible by a prime exceeding $k$ and $P(b) \leqslant k$, we have $n+(k-1)$ $d>k^{\ell}$. For $k \geqslant 4$, we see from [SS01, Theorem $4^{\prime}$ ] and [SST02, Theorem 1] that $\Delta$ is divisible by at least $\chi_{0}=\left[\frac{1}{5} \pi(k)\right]+2$ primes exceeding $k$, except when $(n, d, k)$ equals one of the tuples $(1,5,4)$, $(2,7,4),(3,5,4),(1,2,5),(2,7,5),(4,7,5),(4,23,5)$. We check that these values of $(n, d, k)$ do not satisfy (1.1). Thus, for $k \geqslant 4$ we conclude that $\Delta$ is always divisible by at least $\chi_{0}$ primes $>k$. Therefore, we see from (1.1) that

$$
n+(k-1) d \geqslant q_{\chi_{0}}^{\ell}(k) \text { for } k \geqslant 4 .
$$

Hence, from (1.3), we get $\delta>1 / k$. Furthermore, we derive from (1.1) that

$$
n+i d=a_{i} x_{i}^{\ell}, \quad P\left(a_{i}\right) \leqslant k, \quad a_{i} \text { is } \ell \text { th power free for } 0 \leqslant i<k
$$

and

$$
n+i d=A_{i} X_{i}^{\ell}, \quad P\left(A_{i}\right) \leqslant k, \quad \operatorname{gcd}\left(\prod p, X_{i}\right)=1 \quad \text { for } 0 \leqslant i<k
$$

where the product $\prod p$ is taken over all primes $p$ with $p \leqslant k$.

We say that an integer $N \geqslant 1$ has Property $P_{0}$ if all the prime factors of $N$ which are greater than $k$ divide it to an order $\equiv 0(\bmod \ell)$. From $(2.8)$ and $(2.9)$, we see that every term of $\Delta$ has Property $P_{0}$. Suppose that $N_{1}$ and $N_{2}$ are integers satisfying $0<N_{1}<N_{2}$. Let $r \geqslant 1$ and $t$ be integers. We set

$$
M_{r, t}=M_{r, t}\left(N_{1}, N_{2}\right)=N_{1}\left(1-\frac{t}{r}\right)+N_{2} \frac{t}{r}
$$

We say that the triple $\left(N_{1}, N_{2}, r\right)$ has Property $P_{1}$ if (i) or (ii) given below holds according as $r>1$ or $r=1$, respectively.

(i) Let $r>1$. Then $\left(N_{2}-N_{1}\right) / r$ is an integer and $M_{r, t}$ has Property $P_{0}$ for every $t$ with $0 \leqslant t \leqslant r$.

(ii) Let $r=1$. Then either $M_{1, t}$ with $0 \leqslant t \leqslant\left[\frac{k}{2}\right]$ has Property $P_{0}$ or $M_{1, t}$ with $-\left[\frac{k}{2}\right] \leqslant t \leqslant 0$ has Property $P_{0}$.

Suppose that $N_{1}=n+i d, N_{2}=n+j d$ with $0 \leqslant i<j<k$, then $\left(N_{2}-N_{1}\right) /(j-i)=d$ and $M_{j-i, t}$ with $0 \leqslant t \leqslant j-i$ is a term of the product $\Delta$ and hence has Property $P_{0}$. Therefore (i) is satisfied. Similarly (ii) is also satisfied. Thus, the triple $\left(N_{1}, N_{2}, j-i\right)$ has Property $P_{1}$. We say that the triple $\left(N_{1}, N_{2}, r\right)$ has Property $P_{2}$ if $\left(N_{2}-N_{1}\right) / r$ is an integer and is divisible by a prime $\equiv 1$ $\left(\bmod \ell^{\prime}\right)$ for every odd prime $\ell^{\prime}$ dividing $\ell$. By Lemma 9 , we see that if $k \geqslant 4$ and $N_{1}=n+i d$, $N_{2}=n+j d$ with $0 \leqslant i<j<k$, then the triple $\left(N_{1}, N_{2}, j-i\right)$ has Property $P_{2}$.

Let $S=\left\{A_{0}, \ldots, A_{k-1}\right\}$ and $T=\left\{a_{0}, \ldots, a_{k-1}\right\}$. Furthermore, let $I=\left\{\mu \mid X_{\mu} \neq 1\right.$ with $0 \leqslant \mu<k\}$ and let $S_{1}$ be the set of $A_{\mu} \in S$ with $\mu \in I$. As already mentioned, we assume that $|I| \geqslant 1$. Suppose that $m_{1} \geqslant 1$ and $m_{2} \geqslant 0$ are integers such that $m_{1}+m_{2} \leqslant \pi(k)$. Now we state some counting functions, which were first introduced in Erdös and Selfridge [ES75, pp. 297-299] for the case of consecutive integers. Let $H\left(d, k, m_{1}, m_{2}\right)$ denote the number of distinct $a_{i}$ in $T$ that are composed only of $q_{1}, \ldots, q_{m_{1}}$ and divisible by at most one of the primes $q_{m_{1}+1}, \ldots, q_{m_{1}+m_{2}}$ 


\section{N. Saradha And T. N. Shorey}

that divides at most to the first power. In particular, when $m_{2}=0$, we see that $H\left(d, k, m_{1}, 0\right)$ denotes the number of distinct $a_{i}$ in $T$ that are composed only of $q_{1}, \ldots, q_{m_{1}}$. It can be seen that

$$
H\left(d, k, m_{1}, m_{2}\right) \geqslant|T|-\sum_{i=m_{1}+m_{2}+1}^{\pi(k)}\left(\left[\frac{k}{q_{i}}\right]+\epsilon_{i}^{\prime}\right)-\sum_{m_{1}+1 \leqslant j \leqslant h \leqslant m_{1}+m_{2}}\left(\left[\frac{k}{q_{j} q_{h}}\right]+\epsilon_{j h}^{\prime}\right)
$$

where $\epsilon_{i}^{\prime}=0$ if $q_{i} \mid k$ or $q_{i}>k, \epsilon_{i}^{\prime}=1$ otherwise and $\epsilon_{j h}^{\prime}=1$ if $q_{j} q_{h} \nmid k$ and $q_{j}, q_{h} \leqslant k, \epsilon_{j h}^{\prime}=0$ otherwise. We define $\epsilon_{i}=0$ if $p_{i} \mid k$ and $\epsilon_{i}=1$ otherwise; $\epsilon_{j h}=0$ if $p_{j} p_{h} \mid k$ and $\epsilon_{j h}=1$ otherwise. Then we find that

$$
\left[\frac{k}{q_{i}}\right]+\epsilon_{i}^{\prime} \leqslant\left[\frac{k}{p_{i}}\right]+\epsilon_{i} \text { and }\left[\frac{k}{q_{j} q_{h}}\right]+\epsilon_{j h}^{\prime} \leqslant\left[\frac{k}{p_{j} p_{h}}\right]+\epsilon_{j h} .
$$

For showing the first inequality, we may assume that $\epsilon_{i}^{\prime}=1, \epsilon_{i}=0$ implying that $q_{i}>p_{i}, p_{i} \mid k$ and the assertion follows. The proof for the second inequality is similar. Hence we get from (2.11) that

$$
H\left(d, k, m_{1}, m_{2}\right) \geqslant H_{0}^{\prime}\left(k, m_{1}, m_{2}\right)
$$

where

$$
H_{0}^{\prime}\left(k, m_{1}, m_{2}\right)=|T|-\sum_{i=m_{1}+m_{2}+1}^{\pi(k)}\left(\left[\frac{k}{p_{i}}\right]+\epsilon_{i}\right)-\sum_{m_{1}+1 \leqslant j \leqslant h \leqslant m_{1}+m_{2}}\left(\left[\frac{k}{p_{j} p_{h}}\right]+\epsilon_{j h}\right) .
$$

In particular, we have

$$
H\left(d, k, m_{1}, 0\right) \geqslant H_{0}^{\prime}\left(k, m_{1}, 0\right)=|T|-\sum_{i=m_{1}+1}^{\pi(k)}\left(\left[\frac{k}{p_{i}}\right]+\epsilon_{i}\right) .
$$

We use the above inequality for $k \leqslant 2957$. If $k>2957$, we use $H\left(d, k, m_{1}, m_{2}\right)$ and $H_{0}^{\prime}\left(k, m_{1}, m_{2}\right)$ with $m_{2}>0$. When $m_{2}>0$, we take $m_{1}$ and $m_{2}$ such that $p_{1}<\cdots<p_{m_{1}} \leqslant k^{3 / 10}<p_{m_{1}+1}<\cdots<$ $p_{m_{1}+m_{2}} \leqslant \sqrt{k}$. From $(2.13)$ we then derive that

$$
H_{0}^{\prime}\left(k, m_{1}, m_{2}\right) \geqslant H_{0}^{\prime \prime}\left(k, m_{1}, m_{2}\right)
$$

where

$$
H_{0}^{\prime \prime}\left(k, m_{1}, m_{2}\right):=|T|-\sum_{\sqrt{k}<p \leqslant k}\left(\left[\frac{k}{p}\right]+1\right)-\frac{k}{2}\left(\sum_{i=1}^{m_{2}} \frac{1}{p_{m_{1}+i}^{2}}+\left(\sum_{i=1}^{m_{2}} \frac{1}{p_{m_{1}+i}}\right)^{2}\right)-\left(\begin{array}{c}
m_{2}+1 \\
2
\end{array}\right) .
$$

By combining (2.12) and (2.15), we get

$$
H\left(d, k, m_{1}, m_{2}\right) \geqslant H_{0}^{\prime \prime}\left(k, m_{1}, m_{2}\right) .
$$

Let $|T|=k$, i.e. all $a_{i}$ are distinct. In Table 1 , we display a lower bound $H_{1}\left(m_{1}\right)$ for $H_{0}^{\prime}\left(k, m_{1}, 0\right)$ given by (2.14) when $k$ varies over an interval and $m_{1}$ is suitably chosen. In Table 2 , we display a lower bound $H_{2}\left(m_{1}, m_{2}\right)$ for $H_{0}^{\prime \prime}\left(k, m_{1}, m_{2}\right)$ given by $(2.16)$ when $k$ varies over an interval and $m_{1}, m_{2}$ are suitably chosen.

By Table 1 and (2.14), we have $H\left(d, k, m_{1}, 0\right) \geqslant 4$ for $k=23,24$. We sharpen this as $H\left(d, k, m_{1}, 0\right)$ $\geqslant 5$ for $k=23,24$. Let $k=24$. Suppose that $H\left(d, k, m_{1}, 0\right)=4$. This means that the number of $a_{i}$ that the primes $23,19,17,13,11,7,5$ divide is given by $2,2,2,2,3,4,5$, respectively, and no two primes divide the same $a_{i}$. This implies that 23 divides $a_{0}, a_{23}$. Then it is impossible that 11 divides three different $a_{i}$. The argument for $k=23$ is similar. For $m_{1}>0$ and $\alpha_{i}>0$ with $1 \leqslant i \leqslant m_{1}$, we also need the following counting function. Let $G\left(d, k, m_{1}, \alpha_{1}, \ldots, \alpha_{m_{1}}\right)$ denote the number of $A_{j}$ in $S$ that are composed of $q_{1}, \ldots, q_{m_{1}}$ and $\operatorname{ord}_{q_{i}}\left(A_{j}\right) \leqslant \alpha_{i}-1$ for $1 \leqslant i \leqslant m_{1}$. 


\section{PERFECT POWERS IN ARITHMETIC PROGRESSION}

TABLE 1.

\begin{tabular}{cccccc}
\hline$m_{1}$ & Range of $k$ & $H_{1}\left(m_{1}\right)$ & $m_{1}$ & Range of $k$ & $H_{1}\left(m_{1}\right)$ \\
\hline 2 & $4 \leqslant k \leqslant 10$ & 4 & 5 & $286 \leqslant k \leqslant 600$ & 27 \\
2 & $11 \leqslant k \leqslant 22$ & 5 & 6 & $601 \leqslant k \leqslant 1097$ & 45 \\
2 & 23,24 & 4 & 7 & $1098 \leqslant k \leqslant 1669$ & 77 \\
3 & $25 \leqslant k \leqslant 90$ & 10 & 8 & $1670 \leqslant k \leqslant 2478$ & 132 \\
4 & $91 \leqslant k \leqslant 285$ & 16 & 9 & $2479 \leqslant k \leqslant 2957$ & 227 \\
\hline
\end{tabular}

TABLE 2 .

\begin{tabular}{cccccccc}
\hline$m_{1}$ & $m_{2}$ & Range of $k$ & $H_{2}\left(m_{1}, m_{2}\right)$ & $m_{1}$ & $m_{2}$ & Range of $k$ & $H_{2}\left(m_{1}, m_{2}\right)$ \\
\hline 4 & 12 & $2958-2960$ & 271 & 6 & 15 & $5329-6240$ & 832 \\
5 & 11 & $2961-3480$ & 420 & 6 & 16 & $6241-6888$ & 917 \\
5 & 12 & $3481-3720$ & 465 & 6 & 17 & $6889-7920$ & 986 \\
5 & 13 & $3721-4488$ & 494 & 6 & 18 & $7921-9408$ & 1071 \\
5 & 14 & $4489-5040$ & 545 & 6 & 19 & $9409-10200$ & 1171 \\
5 & 15 & $5041-5165$ & 582 & 6 & 20 & $10201-10608$ & 1237 \\
6 & 14 & $5166-5328$ & 792 & 6 & 21 & $10609-11379$ & 1285 \\
\hline
\end{tabular}

Then $G\left(d, k, m_{1}, \alpha_{1}, \ldots, \alpha_{m_{1}}\right) \geqslant G_{0}\left(k, m_{1}, \alpha_{1}, \ldots, \alpha_{m_{1}}\right)$ where

$$
G_{0}=G_{0}\left(k, m_{1}, \alpha_{1}, \ldots, \alpha_{m_{1}}\right)=|S|-\sum_{i=1}^{m_{1}}\left(\left[\frac{k}{p_{i}^{\alpha_{i}}}\right]+\epsilon_{i}^{\prime \prime}\right)-\sum_{i=m_{1}+1}^{\pi(k)}\left(\left[\frac{k}{p_{i}}\right]+\epsilon_{i}\right)
$$

with $\epsilon_{i}$ as defined earlier and $\epsilon_{i}^{\prime \prime}=0$ if $p_{i}^{\alpha_{i}} \mid k, \epsilon_{i}^{\prime \prime}=1$ otherwise.

We conclude this section with a lemma which is useful for computation.

Lemma 1. Suppose that $k_{1}$ and $k_{2}$ are two consecutive primes and let $k^{\prime}$ be an integer with $k_{1}<$ $k^{\prime}<k_{2}$. Suppose that (1.1) does not hold for $k=k_{1}$. Then (1.1) does not hold for $k=k^{\prime}$.

Proof. Suppose that (1.1) holds for $k=k^{\prime}$. Since $k_{1}<k^{\prime}<k_{2}$ and $k_{1}, k_{2}$ are consecutive primes, $P(b) \leqslant k^{\prime}$ implies that $P(b) \leqslant k_{1}$. Let $k^{\prime}=k_{1}+h$. By deleting the terms $n+\left(k^{\prime}-1\right) d, \ldots, n+\left(k^{\prime}-h\right) d$, we see from $(2.8)$ that

$$
n(n+d) \cdots\left(n+\left(k_{1}-1\right) d\right)=b^{\prime} y_{1}^{\ell}, \quad P\left(b^{\prime}\right) \leqslant k_{1}
$$

for some positive integers $b^{\prime}$ and $y_{1}$. Thus (1.1) holds with $k=k_{1}$, a contradiction.

For the proof of Theorem 1, we see from Lemma 1 that it suffices to show that (1.1) does not hold under Hypothesis A.

\section{An iterative procedure to improve the lower bound for $n+(k-1) d$}

In this section, we give an iterative procedure in Lemma 3 by which we improve the lower bound for $n+(k-1) d$ given by $(2.7)$ and [SS01, Lemma 3]. This procedure is an analogue of that given for the case $\ell=2$ in [SS03]. We first give a lemma in which we estimate the number of elements of $I$.

Lemma 2. Let $k \geqslant 4$. Then (1.1) implies that

$$
|I|>k-\frac{(k-1) \log (k-1)+\log \beta}{\log d+\log (k-1)}-\pi_{d}(k)-1
$$




\section{N. Saradha And T. N. Shorey}

and

$$
|I|>k-\frac{(k-1) \log (k-1)+\log \beta}{\log n_{0}}-\pi_{d}(k)-\phi
$$

where $n_{0}=\max (n, 3), \phi=1$ if $n=1,2$ and $\phi=0$ if $n>2$.

The proof is similar to [SS03, Lemma 3]. We require $\pi_{d}(k)$ instead of $\pi_{d}(k-1)$ in [SS03, Lemma 3] since we now have $P\left(A_{i}\right) \leqslant k$. In the next lemma, we describe the iterative procedure.

Lemma 3. Assume (1.1) such that all $A_{j}$ given by (2.9) are distinct. Then the following assertions hold.

(i) We have

$$
n+(k-1) d \geqslant \max \left(\beta_{3}\left(1, \eta_{1}\right) p_{\pi(k)+1}^{\ell}, p_{\pi(k)+\beta_{3}\left(1, \eta_{1}\right)}^{\ell}\right)
$$

where $\eta_{1}=0$ if $d<k^{\ell-1} / 2$ and $\eta_{1}=1$ otherwise.

(ii) Let $n+(k-1) d \geqslant G_{1} k^{\ell+1}$ with $G_{1} \geqslant 1 / k$. For $i \geqslant 2$, define

$$
g_{i} k^{\ell+1} \leqslant \beta_{5}\left(G_{i-1}, \eta_{i}\right) p_{\pi(k)+1}^{\ell}, \quad G_{i}=\max \left(G_{i-1}, g_{i}\right)
$$

where $\eta_{i}=0$ if $d<G_{i-1} k^{\ell} / 2$ and $\eta_{i}=1$ otherwise. Then $n+(k-1) d \geqslant G_{i} k^{\ell+1}$.

(iii) Let $i_{0}$ be fixed with $n+(k-1) d \geqslant G_{i_{0}} k^{\ell+1}$ and $\eta_{i_{0}+1}=\eta_{1}^{\prime}$. Let

$$
h_{1}=\frac{\beta_{5}\left(G_{i_{0}}, \eta_{i_{0}+1}\right)}{k}, \quad h^{\prime \prime}<h_{1}, v_{1} \leqslant \frac{\left(\left[h^{\prime \prime} k\right]+1\right) p_{h_{1} k-\left[h^{\prime \prime} k\right]+\pi(k)}^{\ell}}{k^{\ell+1}} .
$$

Then $n+(k-1) d \geqslant V_{1} k^{\ell+1}$ where $V_{1}=\max \left(G_{i_{0}}, v_{1}\right)$.

(iv) For $i \geqslant 2$, we define

$$
v_{i} \leqslant \frac{\left(\left[h^{\prime \prime} k\right]+1\right) p_{h_{i} k-\left[h^{\prime \prime} k\right]+\pi(k)}^{\ell}}{k^{\ell+1}}, \quad V_{i}=\max \left(V_{i-1}, v_{i}\right)
$$

where

$$
h_{i}=\frac{\beta_{5}\left(V_{i-1}, \eta_{i}^{\prime}\right)}{k} \quad \text { with } \eta_{i}^{\prime}= \begin{cases}0 & \text { if } d<V_{i-1} k^{\ell} / 2 \\ 1 & \text { otherwise. }\end{cases}
$$

Then $n+(k-1) d \geqslant V_{i} k^{\ell+1}$.

Proof. (i) Suppose that $d \geqslant k^{\ell-1} / 2$. Then we use (3.1) to estimate $|I|$. If $d<k^{\ell-1} / 2$, then we use (2.7) to find $n>k^{\ell} / 2$, which we use in (3.2) to estimate $|I|$. Thus we get $|I|>\beta_{2}\left(1, \eta_{1}\right)$, which, together with $|I| \geqslant 1$, implies that $|I| \geqslant \beta_{3}\left(1, \eta_{1}\right)$. We arrange all the $X_{j}$ with $j \in I$ in increasing order. Since these $X_{j}$ are all distinct, we have $n+(k-1) d \geqslant p_{\pi(k)+\beta_{3}\left(1, \eta_{1}\right)}^{\ell}$. Since $A_{j}$ are distinct, we have $\left|S_{1}\right|=|I| \geqslant \beta_{3}\left(1, \eta_{1}\right)$. Now we arrange these $A_{j}$ in $S_{1}$ in increasing order and observe that each of the corresponding $X_{j}$ has a prime factor greater than $k$. This gives $n+(k-1) d \geqslant \beta_{3}\left(1, \eta_{1}\right) p_{\pi(k)+1}^{\ell}$, which proves (i).

(ii) Let $n+(k-1) d \geqslant G_{1} k^{\ell+1}$. Note that $n+(k-1) d \geqslant G_{1} k^{\ell+1}$ with $G_{1}=1 / k$ is satisfied by (2.7). We prove the assertion for $i=2$. We use (3.1) if $d \geqslant G_{1} k^{\ell} / 2$ and if otherwise, we see that $n \geqslant G_{1} k^{\ell+1} / 2$ and we use (3.2) to estimate $|I|$. Hence $\left|S_{1}\right|=|I| \geqslant \beta_{5}\left(G_{1}, \eta_{2}\right)$, which implies that $n+(k-1) d \geqslant g_{2} k^{\ell+1}$. Thus $n+(k-1) d \geqslant G_{2} k^{\ell+1}$. The assertion for $i \geqslant 3$ follows similarly.

(iii) Let $n+(k-1) d \geqslant G_{i_{0}} k^{\ell+1}$. We proceed as in (ii) to get $\left|S_{1}\right| \geqslant \beta_{5}\left(G_{i_{0}}, \eta_{i_{0}+1}\right)$. Thus there are at least $h_{1} k$ distinct $A_{j}$ with $j \in I$. We arrange them in increasing order and remove the first $\left[h^{\prime \prime} k\right]$ of these $A_{j}$. Thus we are left with $h_{1} k-\left[h^{\prime \prime} k\right] \geqslant 1$ of the $A_{j}$ each of which exceeds $\left[h^{\prime \prime} k\right]+1$ and the largest $X_{j}$ is divisible by a prime greater than or equal to $p_{h_{1} k-\left[h^{\prime \prime} k\right]+\pi(k)}$. Now the assertion follows immediately. 


\section{PERFECT POWERS IN ARITHMETIC PROGRESSION}

(iv) We have $n+(k-1) d \geqslant V_{1} k^{\ell+1}$ by (iii). Hence we get $\left|S_{1}\right| \geqslant \beta_{5}\left(V_{1}, \eta_{2}^{\prime}\right)$. Thus there are at least $h_{2} k$ distinct $A_{j}$ with $j \in I$. Furthermore, $\beta_{5}(s, h)$ is a non-decreasing function of $s$. Hence $h_{2} \geqslant h_{1}$. Now we proceed as in (iii) to get $n+(k-1) d \geqslant\left(\left[h^{\prime \prime} k\right]+1\right) p_{h_{2} k-\left[h^{\prime \prime} k\right]+\pi(k)}^{\ell}$. Hence $n+(k-1) d \geqslant$ $\max \left(V_{1}, v_{2}\right) k^{\ell+1}$. This proves the assertion for $i=2$. The assertion for $i \geqslant 3$ follows similarly.

We illustrate Lemma 3 by means of an example. In this example and at all places in the paper where we compute $\delta$, we take $h^{\prime \prime}=0.16$ and we iterate four times. The value of $h^{\prime \prime}$ is not optimal for every $k$. Furthermore, in this example, we apply Lemma 3 with $d \leqslant k^{\ell-1} / 2$ so that $\eta_{i}=\eta_{i}^{\prime}=0$ for $1 \leqslant i \leqslant 4$.

Example. We show that

$$
\delta \geqslant 6.0048 k^{\ell+1} \text { for } k \geqslant 173 \text { and } \ell \geqslant 3 .
$$

We observe that the functions $\beta_{3}$ and $\beta_{5}$ given by (2.4) and (2.6) are non-decreasing functions of $\ell$ and $k$. Hence, while evaluating these functions in this example, it is enough to evaluate them at $k=173$ and $\ell=3$. We first take $k=173$. By using exact values of $\pi(k)$, we find that $\beta_{3}(1,0) \geqslant 73$. Now using exact values for $p_{i}$, by Lemma $3(\mathrm{i})$ we get $n+(k-1) d \geqslant \max \left(73 \times 179^{\ell}, 617^{\ell}\right) \geqslant$ $0.4674 k^{\ell+1}$. In Lemma $3(\mathrm{ii})$, we take $G_{1}=0.4674$. We compute $g_{2}=0.5570$. Thus $G_{2}=0.5570$. Similarly we find $G_{3}=G_{4}=0.5634$. In Lemma 3(iii), we take $i_{0}=4$. We get $h_{1}>0.5>0.16=h^{\prime \prime}$. Hence $v_{1}=5.1160$. Thus $V_{1}=5.1160$. In Lemma $3(\mathrm{iv})$, we compute $v_{2}=5.8194$. Hence $V_{2}=5.8194$. Similarly we find $V_{3}=V_{4}=6.0048$. Thus we obtain (3.3).

Now let $k \geqslant 3000$. In Lemma 3 we use the approximate values for $p_{i}$ and $\pi(k)$ given by $(2.1)$. We also use $p_{\pi(k)+1}>k$. Thus we find by Lemma $3(\mathrm{i})$ that $n+(k-1) d \geqslant \rho_{1} k^{\ell+1}$ where

$$
\rho_{1}=1-\frac{\log (k-1)}{\ell \log (k-1)-\log 2}-\frac{1}{\log k}-\frac{1.5}{\log ^{2} k}-\frac{1}{k} .
$$

We observe that $\rho_{1}$ increases as $k$ and $\ell$ increase. We compute $\rho_{1}$ at $k=3000$ and $\ell=3$ to get $n+(k-1) d \geqslant 0.5081 k^{\ell+1}$. Thus $n+(k-1) d \geqslant 0.5081 k^{\ell+1}$ for all $k \geqslant 3000$ and $\ell \geqslant 3$. Next we apply the iterative procedure of Lemma 3(ii). We take $G_{1}=\rho_{1}$. Then for $i \geqslant 2$, we get $n+(k-1) d \geqslant \rho_{i} k^{\ell+1}$ where

$$
\rho_{i}=1-\frac{\log (k-1)}{(\ell+1) \log (k-1)+\log \rho_{i-1}-\log 2}-\frac{1}{\log k}-\frac{1.5}{\log ^{2} k}-\frac{1}{k} .
$$

We observe that $\rho_{i}$ is an increasing function of $k$ and $\ell$. We compute $\rho_{2}=0.5901, \rho_{3}=\rho_{4}=0.5914$. Thus $n+(k-1) d \geqslant 0.5914 k^{\ell+1}$ for all $k \geqslant 3000$ and $\ell \geqslant 3$. Finally, we apply the iterative procedure of Lemma 3(iii), (iv). We take $G_{4}=0.5914$ and $h^{\prime \prime}=0.16$. We set $\rho_{0}^{\prime}=0.5914$. Then for $i \geqslant 1$ we get $n+(k-1) d \geqslant \rho_{i}^{\prime} k^{\ell+1}$ where

$$
\rho_{i}^{\prime}=h^{\prime \prime}\left(h_{i}^{\prime}-h^{\prime \prime}\right)^{\ell} \log ^{\ell}\left(h_{i}^{\prime} k-h^{\prime \prime} k+\frac{k}{\log k}\right)
$$

with

$$
h_{i}^{\prime}=1-\frac{\log (k-1)}{(\ell+1) \log (k-1)+\log \rho_{i-1}^{\prime}-\log 2}-\frac{1}{\log k}-\frac{1.5}{\log ^{2} k}-\frac{1}{k} .
$$

We observe that $\rho_{i}^{\prime}$ is an increasing function of $\ell$ and $k \geqslant 200$ by noticing that $h_{i}^{\prime}>\rho_{1}$ and $\left(h_{i}^{\prime}-h^{\prime \prime}\right) \log \left(h_{i}^{\prime} k-h^{\prime \prime} k+k / \log k\right)>1$ for $k \geqslant 200$. We compute $h_{1}^{\prime}=0.5914, \rho_{1}^{\prime}=5.2507 ; h_{2}^{\prime}=0.6086$, $\rho_{2}^{\prime}=5.9770 ; h_{3}^{\prime}=0.60963, \rho_{3}^{\prime}=6.0191, h_{4}^{\prime}=0.60968, \rho_{4}^{\prime}=6.0213$. Thus we obtain (3.3) for $k \geqslant 3000, l \geqslant 3$. Now for $k$ with $173<k<3000$, we apply Lemma 3 with exact values of $p_{i}$ and $\pi(k)$ as in the case $k=173$ to obtain (3.3). This completes the proof of (3.3).

It is clear from the example above that the lower bound given by Lemma 3 for $\delta$ is a nondecreasing function of $\ell$ and $k$. We use this fact without mentioning it in the following. 


\section{N. Saradha And T. N. Shorey}

\section{Distinctness of $\boldsymbol{A}_{j}$}

A pre-requisite for the iterative procedure in Lemma 3 is that all $A_{j}$ are distinct. In this section, we show that when $A_{j}$ are not all distinct, then we can bound from above $n$ and $k$ in terms of $\ell$ and $d$. These bounds are decreasing functions of $\ell$ and $1 / d$ (see (4.1)). Using these bounds we show in Corollary 1 that $A_{j}$ are distinct whenever (1.1) with Hypothesis A holds.

Lemma 4. Suppose that (1.1) holds with $k \geqslant 4$. Then either $A_{j}$ with $0 \leqslant j<k$ are distinct or

$$
k \leqslant\left(\left(\frac{d}{2 \ell}\right)^{\ell /(\ell-1)}+\frac{d}{k^{1 /(\ell-1)}}\right)^{(\ell-1) /\left(\ell^{2}-2 \ell\right)} \text { and } n<\left(\frac{k d}{2 \ell}\right)^{\ell /(\ell-1)} .
$$

Proof. Suppose that there exist $A_{i}, A_{j}$ with $0 \leqslant j<i<k$ such that

$$
A_{i}=A_{j} .
$$

Then we see from (2.9) that $X_{i} \geqslant X_{j}+2$ and

$$
(k-1) d \geqslant(i-j) d=(n+i d)-(n+j d)=A_{i} X_{i}^{\ell}-A_{j} X_{j}^{\ell} \geqslant 2 \ell A_{j} X_{j}^{\ell-1} .
$$

Thus it follows that $k d>2 \ell\left(A_{j} X_{j}^{\ell}\right)^{(\ell-1) / \ell} \geqslant 2 \ell n^{(\ell-1) / \ell}$. This gives the bound for $n$ in (4.1). Furthermore, we use (2.7) to get

$$
k^{\ell}<q_{\chi_{0}}^{\ell}(k) \leqslant n+(k-1) d<\left(\frac{k d}{2 \ell}\right)^{\ell /(\ell-1)}+k d,
$$

which gives the estimate for $k$ in (4.1).

As a consequence of Lemma 4, we get the following.

Lemma 5. Assume (1.1) with Hypothesis $A$ and $k \geqslant 4$. Suppose that $A_{j}$ are all not distinct. Then

$$
\left\{\begin{array}{l}
\ell=4, k=4 ; \quad \ell=5, k \leqslant 7 ; \quad \ell=6, k=4 ; \quad \ell=7, k \leqslant 11 ; \quad \ell=8, k \leqslant 7 ; \quad \ell=9,10, k=4 ; \\
\ell=11, k \leqslant 19 ; \quad \ell=12, k \leqslant 13 ; \quad \ell=13,14, k \leqslant 7 ; \quad \ell=15, k \leqslant 5 ; \quad \ell=16,17,18, k=4 .
\end{array}\right.
$$

Proof. Assume (1.1) with Hypothesis A and $k \geqslant 4$. Suppose that $A_{j}$ are not all distinct. Then (4.1) and (4.4) are valid. By (4.1), we see that $k \leqslant 5$ for $\ell \geqslant 19$, which we sharpen by (4.4) to $k<4$ for $\ell \geqslant 19$. Therefore $\ell \leqslant 18$. Let $\ell=4$. Then $d \leqslant d_{1}=950$. We use (4.1) to get $k \leqslant 13$. Now for $5 \leqslant k \leqslant 13$, we find that (4.4) is not valid. Thus $k=4$. The bound for $k$ in (4.5) for all other values of $\ell \leqslant 18$ is found in a similar manner.

Now we proceed to exclude all of the values of $\ell$ and $k$ in (4.5). We show the following.

Corollary 1. Assume (1.1) with Hypothesis $A$ and $k \geqslant 4$. Then $A_{j}$ are distinct.

Proof. Assume (1.1) with Hypothesis A and $k \geqslant 4$. Suppose that $A_{j}$ are not all distinct. Then (4.1)-(4.5) are valid. We fix $k, \ell$ where $k, \ell$ are given by (4.5). From (4.4), we see that $n+(k-1) d<\delta_{3}$ where $\delta_{3}$ is a bounded positive number. Let $1 \leqslant r<k$. We take $U_{1}(r)$ to be the set of divisors of $r$. Let $U_{2}$ be the set of all positive integers not exceeding $\delta_{3}^{1 / \ell}$ and having the least prime factor greater than $k$. We always include 1 in $U_{2}$. We form $U_{3}(r)$ to be the set of pairs $\left(h X^{\ell}, h Y^{\ell}\right)$ with $h \in U_{1}(r)$ and $X, Y$ in $U_{2}$ with $X<Y$ and $\operatorname{gcd}(X, Y)=1$. Let $U_{4}$ be the set of triples $\left(h X^{\ell}, h Y^{\ell}, r\right)$ with $\left(h X^{\ell}, h Y^{\ell}\right) \in U_{3}(r), 1 \leqslant r<k$ such that the triple $\left(h X^{\ell}, h Y^{\ell}, r\right)$ has Property $P_{1}$. From (4.2)-(4.4), we find that there exist $0 \leqslant j<i<k$ such that $A_{i}=A_{j}, A_{j} \mid(i-j)$ and $X_{i}, X_{j}$ do not exceed $\delta_{3}^{1 / \ell}$. Also $\operatorname{gcd}\left(X_{i}, X_{j}\right)=1$. Thus $\left(A_{j} X_{j}^{\ell}, A_{j} X_{i}^{\ell}\right) \in U_{3}(r)$ with $r=i-j$. 


\section{PERFECT POWERS IN ARITHMETIC PROGRESSION}

Furthermore, $d=A_{j}\left(X_{i}^{\ell}-X_{j}^{\ell}\right) /(i-j)$, which, by (2.9) and (2.10), implies that

$$
M_{r, t}\left(A_{j} X_{j}^{\ell}, A_{j} X_{i}^{\ell}\right)=A_{j} X_{j}^{\ell}\left(1-\frac{t}{r}\right)+A_{j} X_{i}^{\ell} \frac{t}{r}=A_{j} X_{j}^{\ell}+t d=n+(j+t) d .
$$

If $0 \leqslant t \leqslant r$, we see that the left-hand side is a term of the product $\Delta$ and hence has Property $P_{0}$. Suppose that $r=1$. Then we take $0 \leqslant t \leqslant\left[\frac{k}{2}\right]$ if $0 \leqslant j \leqslant\left[\frac{k}{2}\right]-1$ and $-\left[\frac{k}{2}\right] \leqslant t \leqslant 0$ if $\left[\frac{k}{2}\right] \leqslant j \leqslant k-1$. Then $j+t \leqslant k-1$ in the former case and $j+t \geqslant 0$ in the latter case, implying that $M_{1, t}\left(A_{j} X_{j}^{\ell}, A_{j} X_{i}^{\ell}\right)$ is a term of the product $\Delta$ and therefore has Property $P_{0}$. Thus the triple $\left(A_{j} X_{j}^{\ell}, A_{j} X_{i}^{\ell}, r\right)$ has Property $P_{1}$. Hence $\left(A_{j} X_{j}^{\ell}, A_{j} X_{i}^{\ell}, r\right) \in U_{4}$. On the other hand, given $k, \ell$ as in (4.5), we check that for any pair $\left(h X^{\ell}, h Y^{\ell}\right) \in U_{3}(r)$ with $1<r<k$, Property $P_{0}$ does not hold for $M_{r, 1}\left(h X^{\ell}, h Y^{\ell}\right)$. When $r=1$, we check that $M_{1,1}\left(h X^{\ell}, h Y^{\ell}\right)$ as well as $M_{1,-1}\left(h X^{\ell}, h Y^{\ell}\right)$ do not have Property $P_{0}$. Thus, no triple $\left(h X^{\ell}, h Y^{\ell}, r\right)$ with $\left(h X^{\ell}, h Y^{\ell}\right) \in U_{3}(r), 1 \leqslant r<k$ has Property $P_{1}$. Hence $U_{4}=\emptyset$. This yields a contradiction.

We illustrate the above procedure with an example. Let $\ell=11, k=19$. Then we have $d \leqslant 10^{15}$ and $n+(k-1) d \leqslant 4 \cdot 6 \times 10^{16}$ by (4.4). We form the set $U_{2}=\{1,23,29,31\}$. For each $1 \leqslant r<19$, we construct $U_{3}(r)$. For instance, we have

$$
U_{3}(17)=\left\{\left(1,23^{11}\right),\left(1,29^{11}\right),\left(1,31^{11}\right),\left(23^{11}, 29^{11}\right),\left(23^{11}, 31^{11}\right),\left(29^{11}, 31^{11}\right),\left(17,17 \cdot 23^{11}\right)\right\} .
$$

We check that none of the triples $\left(h X^{\ell}, h Y^{\ell}, r\right)$ such that $\left(h X^{\ell}, h Y^{\ell}\right) \in U_{3}(r)$ with $h \mid r$ for every $1 \leqslant r<19$ has Property $P_{1}$. Thus $U_{4}=\emptyset$, a contradiction. All other possibilities of $\ell$ and $k$ in (4.5) are excluded similarly.

\section{Upper bound for $n+(k-1) d$ when $\ell$ is even}

We use the method of Erdős [Erd39] to derive an upper bound for $n+(k-1) d$. We also refer thereafter to [SS03] for details.

Lemma 6. Suppose that (1.1) is satisfied with $\ell \geqslant 4$ even. Let $h_{0}=h_{0}(k)$ be a positive integer such that $h_{0}=1$ if $4 \leqslant k \leqslant 24 ; h_{0}=2$ if $25 \leqslant k \leqslant 74 ; h_{0}=4$ if $75 \leqslant k \leqslant 159 ; h_{0}=5$ if $k \geqslant 160$. Then $n<k^{2} d^{2} /\left(4 h_{0}\right)$.

Proof. Since $\ell$ is even we may write

$$
n+i d=b_{i} z_{i}^{2} \quad \text { for } 0 \leqslant i<k
$$

where $b_{i}$ are square free with $P\left(b_{i}\right) \leqslant k$. Let $R$ be the set of $b_{i}$. Suppose that $n \geqslant k^{2} d^{2} /\left(4 h_{0}\right)$. First we show that $|R| \geqslant \min \left(k-2 h_{0}+3, k\right)$. We say that an element $b_{j}$ of $R$ has multiplicity $r_{j}$ if $b_{j}=b_{i}$ for $r_{j}$ values of $i$. In particular, if $b_{j}$ has multiplicity 1 , then it means that $b_{j}$ occurs only once and $b_{j}$ is repeated only when it has multiplicity greater than 1 . Suppose that $|R| \leqslant$ $\min \left(k-2 h_{0}+2, k-1\right)$. Then there are at least $\max \left(2,2 h_{0}-1\right)$ of the $b_{i}$ counted with multiplicity that are repeated. Thus there exist $b_{i}, b_{j}$ such that $b_{i}=b_{j}$ with $0 \leqslant i, j<k, i \neq j$. By (5.1) we assume without loss of generality that $z_{i}>z_{j}$ and

$$
k d>(i-j) d \geqslant 2 b_{j}\left(z_{i}-z_{j}\right) z_{j} \geqslant 2 b_{j}^{1 / 2}\left(z_{i}-z_{j}\right)\left(b_{j} z_{j}^{2}\right)^{1 / 2} \geqslant 2 b_{j}^{1 / 2}\left(z_{i}-z_{j}\right) n^{1 / 2} .
$$

Hence $n<k^{2} d^{2} /\left(4 b_{j}\left(z_{i}-z_{j}\right)^{2}\right)$. Thus $b_{j}\left(z_{i}-z_{j}\right)^{2}<h_{0}$, implying that $h_{0}>1, b_{j}=z_{i}-z_{j}=1$ if $h_{0}=2, b_{j} \in\{1,2,3\}, z_{i}-z_{j}=1$ if $h_{0}=4$ and $b_{j}=1, z_{i}-z_{j}=1,2 ; b_{j} \in\{2,3\}, z_{i}-z_{j}=1$ if $h_{0}=5$, by noting that $b_{j}$ are square free. Therefore, there are at most $2 h_{0}-2$ of the $b_{j}$ counted with multiplicity that are repeated. This is a contradiction since $\max \left(2,2 h_{0}-1\right)=2 h_{0}-1$ by $h_{0}>1$. Thus we have $|R| \geqslant k-2 h_{0}+3$. 


\section{N. Saradha And T. N. Shorey}

Since $b_{i}$ are square free, we follow the argument of [SS03, (6.7), (6.9)] for $k-2 h_{0}+3 \geqslant 63$ to get

$$
(1.5)^{k-2 h_{0}+3}\left(k-2 h_{0}+3\right) ! \leqslant \prod_{i=0}^{k-2 h_{0}+4} b_{i} \leqslant 52 k^{8}(k-1) !(1.0731)^{k} .
$$

This implies that $k \leqslant 260$. Now we use the counting argument as in [SS03]. We explain with an example. Let $k=260$. Then $h_{0}=5$ and $|R| \geqslant k-7$. We find that the number of distinct $b_{i}$ composed of $2,3,5,7,11$ is at least 37 . On the other hand, since $b_{i}$ are square free, this number is at most 32 . This is a contradiction. Thus $k \neq 260$. We exclude all $k$ with $160 \leqslant k<260$ by the above argument. When $75 \leqslant k \leqslant 159$, we have $|R| \geqslant k-5$ and we see that the number of distinct $b_{i}$ composed of 2 , $3,5,7$ exceed 16 , which is a contradiction. Next, when $25 \leqslant k \leqslant 74$, we have $|R| \geqslant k-1$ and we find that the number of distinct $b_{i}$ composed of $2,3,5$ exceed eight giving a contradiction. Finally, when $4 \leqslant k \leqslant 24$, all $b_{j}$ are distinct and by counting the $b_{i}$ composed of 2,3 , we get $k \leqslant 8$. Let $k=8$. Then we see that $b_{2}, b_{3}, b_{4}, b_{5}$ are distinct and they are composed of 2 and 3 . Therefore, these four $b_{i}$ must take all the values of $\{1,2,3,6\}$. Hence, the product of the corresponding terms in $\Delta$ must be a square. A result of Euler states that a product of four terms in an arithmetic progression is never a square. Dickson [Dic52, p. 635] gave a historical reference to Euler's result. We refer to [MS03] for a proof. Thus $k \neq 8$. Similarly, we see that $k \neq 4,6$. Let $k=7$. We have either 5 dividing $b_{0}$ and $b_{5}$ or 5 dividing $b_{1}$ and $b_{6}$. Suppose that 5 divides $b_{0}$ and $b_{5}$. Then 7 divides one of $b_{1}, b_{2}, b_{3}, b_{4}$ by the result of Euler stated above. Suppose that 7 divides $b_{2}$ or $b_{3}$. Since $\left(\frac{b_{1}}{5}\right)=\left(\frac{b_{4}}{5}\right)=\left(\frac{b_{6}}{5}\right)$, we find that $b_{1}, b_{4}, b_{6}$ take values from $\{1,6\}$ or $\{2,3\}$, which is not possible since $b_{i}$ are distinct. Thus 7 divides $b_{1}$ or $b_{4}$. If 7 divides $b_{1}$, then $\left(\frac{b_{2}}{7}\right)=\left(\frac{b_{3}}{7}\right)$ and $\left(\frac{b_{4}}{7}\right)=\left(\frac{b_{6}}{7}\right)$ implying that either $b_{2}, b_{3} \in\{3,6\}$ or $b_{4}, b_{6} \in\{3,6\}$, which is not possible. Likewise 7 dividing $b_{4}$ is excluded. The argument for the case 5 dividing $b_{1}, b_{6}$ is similar. Thus $k \neq 7$. Let $k=5$. Then 5 divides one of $b_{1}, b_{2}, b_{3}$. Suppose that $5 \mid b_{2}$. Then $b_{1}, b_{3} \in\{1,6\}, b_{0}, b_{4} \in\{2,3\}$ or $b_{1}, b_{3} \in\{2,3\}, b_{0}, b_{4} \in\{1,6\}$. This is not possible. Thus $5 \nmid b_{2}$. Let $5 \mid b_{1}$. Then $\left(b_{0}, b_{2}, b_{3}, b_{4}\right)=(6,1,3,2)$. Hence $n \equiv 6(\bmod 8)$ and $n+3 d \equiv 3$ $(\bmod 8)$, which imply that $d \equiv 7(\bmod 8)$. Therefore $n+2 d \equiv 4(\bmod 8)$. When $5 \mid b_{3}$, we get $\left(b_{0}, b_{1}, b_{2}, b_{4}\right)=(2,3,1,6)$ and $n+2 d \equiv 4(\bmod 8)$. We consider the case $\left(b_{0}, b_{2}, b_{3}, b_{4}\right)=(6,1,3,2)$. We have $3 \nmid z_{2}$. Suppose that $3 \mid z_{0} z_{3}$. Then we see from $n+2(n+3 d)=3(n+2 d)$ that $2 z_{0}^{2}+2 z_{3}^{2}=z_{2}^{2}$, which is impossible. Hence $3 \nmid z_{0} z_{3}$. Then we see from $(2.8)$ that $\left(a_{0}, a_{2}, a_{3}, a_{4}\right)=(6,4,3,2)$. Thus

$$
n d+6 d^{2}=(n+2 d)(n+3 d)-n(n+4 d)=12\left(\left(x_{2} x_{3}\right)^{\ell}-\left(x_{0} x_{4}\right)^{\ell}\right),
$$

which implies that $x_{2} x_{3}>x_{0} x_{4}$. Hence

$$
n d+6 d^{2}>12 \ell\left(x_{0} x_{4}\right)^{\ell-1}=12^{1 / \ell} \ell\left(12 x_{0}^{\ell} x_{4}^{\ell}\right)^{(\ell-1) / \ell}>12^{1 / \ell} \ell n^{2(\ell-1) / \ell} .
$$

Thus

$$
n^{(\ell-2) / \ell}<\frac{1}{12^{1 / \ell} \ell}\left(d+\frac{6 d^{2}}{n}\right)<\frac{1}{12^{1 / \ell} \ell}(d+1)
$$

since $n \geqslant 25 d^{2} / 4$. Thus $n<d^{\ell /(\ell-2)} \leqslant d^{2}$, a contradiction. The argument for the case $\left(b_{0}, b_{2}, b_{3}, b_{4}\right)=$ $(2,3,1,6)$ is similar.

\section{Upper bound for $n+(k-1) d$ when $\ell$ is odd}

We assume in this section that $\ell$ is odd and we write $\ell=\ell_{1}^{e_{1}} \cdots \ell_{r}^{e_{r}}$ where $\ell_{i}$ are distinct primes and $e_{i}$ are positive integers. We find an upper bound for $n+(k-1) d$ in Lemma 12 below, which is based on an extension of a result of Erdös and Selfridge on the distinctness of $a_{i}$ and their products. See [ES75, Lemma 1] and [SS01, Lemmas 5 and 6]. We use the following well-known result on cyclotomic polynomials in this extension (see [Ste75]). 


\section{PERFECT POWERS IN ARITHMETIC PROGRESSION}

Lemma 7. For any integer $m>2$ and relatively prime integers $X, Y$ with $X>Y>0$, let $\phi_{m}(X, Y)$ denote the $m$ th cyclotomic polynomial. Then the prime divisors of $\phi_{m}(X, Y)$ are congruent to 1 $(\bmod m)$ except possibly $P(m)$ dividing it at most to the first power.

As a consequence of Lemma 7, we get the following.

Lemma 8. Suppose that $Z_{1}>Z_{2}>0$ are integers with $\operatorname{gcd}\left(Z_{1}, Z_{2}\right)=1$ and

$$
\Phi_{\ell}\left(Z_{1}, Z_{2}\right)=\frac{Z_{1}^{\ell}-Z_{2}^{\ell}}{Z_{1}-Z_{2}}
$$

Then every prime factor $p$ of $\Phi_{\ell}\left(Z_{1}, Z_{2}\right)$ is either congruent to $1\left(\bmod \ell_{i}\right)$ for some $i$ with $1 \leqslant i \leqslant r$ or $p=\ell_{j}$ for some $j$ with $1 \leqslant j \leqslant r$. Furthermore, in the latter case, $\ell_{j}^{e_{j}} \| \Phi_{\ell}\left(Z_{1}, Z_{2}\right)$ provided $\ell_{j}$ is not congruent to $1\left(\bmod \ell_{i}\right)$ for any $\ell_{i}$ with $1 \leqslant i \leqslant r, i \neq j$.

Proof. First, we consider the case when $\ell$ is a prime power, i.e. $\ell=q^{\alpha}$ for some prime $q$ and $\alpha>0$. Then

$$
\Phi_{q^{\alpha}}\left(Z_{1}, Z_{2}\right)=\frac{Z_{1}^{q^{\alpha}}-Z_{2}^{q^{\alpha}}}{Z_{1}-Z_{2}}=\phi_{q}\left(Z_{1}, Z_{2}\right) \phi_{q}\left(Z_{1}^{q}, Z_{2}^{q}\right) \cdots \phi_{q}\left(Z_{1}^{q^{\alpha-1}}, Z_{2}^{q^{\alpha-1}}\right) .
$$

Hence, by Lemma 7 , we see that every prime factor of $\Phi_{q^{\alpha}}\left(Z_{1}, Z_{2}\right)$ is congruent to $1(\bmod q)$ except perhaps $q$. When $q$ divides any of the above cyclotomic polynomials, it divides each of them to the first power. Hence $q^{\alpha} \| \Phi_{q^{\alpha}}\left(Z_{1}, Z_{2}\right)$.

Now we consider any $\ell$. We put $Z_{1,0}=Z_{1} ; Z_{2,0}=Z_{2}$; for $i \geqslant 1, Z_{1, i}=Z_{1, i-1}^{\ell_{i}^{e_{i}}}, Z_{2, i}=Z_{2, i-1}^{\ell_{i}^{e_{i}}}$. Then we have

$$
\Phi_{\ell}\left(Z_{1}, Z_{2}\right)=\Phi_{\ell_{r}^{e_{r}}}\left(Z_{1, r-1}, Z_{2, r-1}\right) \Phi_{\ell_{r-1}^{e_{r-1}}}\left(Z_{1, r-2}, Z_{2, r-2}\right) \cdots \Phi_{\ell_{1}^{e_{1}}}\left(Z_{1,0}, Z_{2,0}\right) .
$$

Now the assertion follows by the case $\ell=q^{\alpha}$ from the previous paragraph. We note here that if $\ell_{i}$ divides $\Phi_{\ell_{i}^{e_{i}}}\left(Z_{1, i-1}, Z_{2, i-1}\right)$ then $\ell_{i}^{e_{i}} \| \Phi_{\ell_{i}^{e_{i}}}\left(Z_{1, i-1}, Z_{2, i-1}\right)$ and $\ell_{i}$ does not divide any other factor whenever $\ell_{i}$ is not congruent to $1\left(\bmod \ell_{j}\right)$ for any $j$ with $1 \leqslant j \leqslant r, j \neq i$. Hence in that case, $\ell_{i}^{e_{i}} \| \Phi_{\ell}\left(Z_{1}, Z_{2}\right)$.

Now we turn to an extension of a result of Erdös and Selfridge on the distinctness of $a_{i}$ and their products. We write $d=D_{1} D_{2}$ where $D_{1}$ is the maximal divisor of $d$ such that every prime divisor of $D_{1}$ is congruent to $1\left(\bmod \ell_{i}\right)$ for some $\ell_{i} \mid \ell$. Thus every prime divisor of $D_{2}$ is incongruent to 1 $\left(\bmod \ell_{i}\right)$ for any $\ell_{i} \mid \ell$. We observe that $D_{1}$ and $D_{2}$ defined here agree with the definitions of $D_{1}$ and $D_{2}$ in [SS01] when $\ell$ is an odd prime. The following has been shown in [SS01].

Lemma 9. Let $\ell$ be an odd prime. Then (1.1) with $k \geqslant 4$ implies that

$$
D_{1}>1 \text {. }
$$

For $i \geqslant 1$, we set $\theta_{i}=1 / \ell_{i}^{\min \left(e_{i}, \operatorname{ord}_{\ell_{i}} D_{2}\right)}$ and

$$
\theta=\theta_{1} \cdots \theta_{r} .
$$

We observe that $\theta=1$ if $\operatorname{gcd}(\ell, d)=1$. We show the following.

Lemma 10. Suppose that (1.1) holds. Let $\ell^{\prime}$ be an integer with $1 \leqslant \ell^{\prime}<\ell$. Furthermore, let

$$
D_{1} \leqslant \frac{\ell \theta}{k \ell^{\prime}} n^{\left(\ell-\ell^{\prime}\right) / \ell}
$$

Then for no distinct $\ell^{\prime}$-tuples $\left(i_{1}, \ldots, i_{\ell^{\prime}}\right)$ and $\left(j_{1}, \ldots, j_{\ell^{\prime}}\right)$ with $i_{1} \leqslant \cdots \leqslant i_{\ell^{\prime}}$ and $j_{1} \leqslant \cdots \leqslant j_{\ell^{\prime}}$, the ratio of the two products $a_{i_{1}} \cdots a_{i_{\ell^{\prime}}}$ and $a_{j_{1}} \cdots a_{j_{\ell^{\prime}}}$ is an $\ell$ th power of a rational number. 


\section{N. Saradha And T. N. Shorey}

Furthermore, for integers $m_{1} \geqslant 1, m_{2} \geqslant 0$ with $m_{1}+m_{2} \leqslant \pi(k)$, we have

$$
\left(\begin{array}{c}
H\left(d, k, m_{1}, m_{2}\right)+\ell^{\prime}-1 \\
\ell^{\prime}
\end{array}\right) \leqslant \ell^{m_{1}}\left(\begin{array}{c}
\ell^{\prime}+m_{2} \\
\ell^{\prime}
\end{array}\right)
$$

where the left-hand side is zero if $H\left(d, k, m_{1}, m_{2}\right)<1$.

Proof. We follow the argument of [SS01, Lemma 5]. Let $\left(i_{1}, \ldots, i_{\ell^{\prime}}\right)$ and $\left(j_{1}, \ldots, j_{\ell^{\prime}}\right)$ with $i_{1} \leqslant \cdots \leqslant$ $i_{\ell^{\prime}}$ and $j_{1} \leqslant \cdots \leqslant j_{\ell^{\prime}}$ be distinct $\ell^{\prime}$-tuples such that

$$
a_{i_{1}} \cdots a_{i_{\ell^{\prime}}}=a_{j_{1}} \cdots a_{{\ell^{\prime}}^{\prime}}\left(\frac{t_{1}}{t_{2}}\right)^{\ell}
$$

where $t_{1}$ and $t_{2}$ are positive integers with $\operatorname{gcd}\left(t_{1}, t_{2}\right)=1$. As in the proof of [SS01, Lemma 5], we may assume that $\left(n+i_{1} d\right) \cdots\left(n+i_{\ell^{\prime}} d\right)>\left(n+j_{1} d\right) \cdots\left(n+j_{\ell^{\prime}} d\right)$ and

$$
\left(n+i_{1} d\right) \cdots\left(n+i_{\ell^{\prime}} d\right)-\left(n+j_{1} d\right) \cdots\left(n+j_{\ell^{\prime}} d\right)=\frac{a_{j_{1}} \cdots a_{{\ell^{\prime}}^{\prime}}}{t_{2}^{\ell}}\left(x^{\ell}-y^{\ell}\right)
$$

where $x=t_{1} x_{i_{1}} \cdots x_{i_{\ell^{\prime}}}, y=t_{2} x_{j_{1}} \cdots x_{{\ell^{\prime}}^{\prime}}$ and $a_{j_{1}} \cdots a_{j_{\ell^{\prime}}} / t_{2}^{\ell}$ is a positive integer. We rewrite (6.5) as

$$
\left(n+i_{1} d\right) \cdots\left(n+i_{\ell^{\prime}} d\right)-\left(n+j_{1} d\right) \cdots\left(n+j_{\ell^{\prime}} d\right)=\frac{a_{j_{1}} \cdots a_{j_{\ell^{\prime}}}}{t_{2}^{\ell}}\left(\frac{x^{\ell}-y^{\ell}}{x-y}\right)(x-y) .
$$

We observe that the left-hand side of (6.6) is divisible by $d$. Also by Lemma 8 and (6.2), we find that $\theta D_{2}$ divides $x-y$ since $\operatorname{gcd}\left(a_{j}, d\right)=1$ for $0 \leqslant j<k$. Thus $x \geqslant y+\theta D_{2}$. We estimate the left-hand side of (6.6) from above and the right-hand side of (6.6) from below as in [SS01, Lemma 6] to obtain

$$
\left\{\left(\begin{array}{l}
\ell \\
1
\end{array}\right) \theta D_{2} n^{\ell^{\prime}(\ell-1) / \ell}-\left(\begin{array}{l}
\ell^{\prime} \\
1
\end{array}\right) k d n^{\ell^{\prime}-1}\right\}+\cdots+\left\{\left(\begin{array}{c}
\ell \\
\ell^{\prime}
\end{array}\right)\left(\theta D_{2}\right)^{\ell^{\prime}} n^{\ell^{\prime}\left(\ell-\ell^{\prime}\right) / \ell}-(k d)^{\ell^{\prime}}\right\}+\cdots+\left(\theta D_{2}\right)^{\ell}<0 .
$$

By (6.3), we see that for each $1 \leqslant i \leqslant \ell^{\prime}$ the term in the $i$ th curly bracket above is positive. This is a contradiction to (6.7). Finally (6.4) follows as an immediate consequence from the argument [ES75, pp. 297-299]. See also [SS01, Lemma 6].

We apply Lemma 10 to get the following.

Lemma 11. Suppose that (1.1) holds with $D_{1} \leqslant(\ell \theta / 2 k) n^{(\ell-2) / \ell}$. Then $k<11380$.

Proof. Suppose that the hypothesis of Lemma 11 is satisfied. Then we observe that (6.3) is satisfied with $\ell^{\prime}=2$. Hence, by Lemma 10, we find that the products $a_{i} a_{j}$ with $i \leqslant j$ are distinct. Then the estimates of [Sar97, Lemma 8] are valid. We use these estimates to conclude $k<11380$ as in [Sar97, pp. 165-166].

Next we apply Lemmas 10 and 11 to bound $n$.

Lemma 12. Suppose that (1.1) holds with $\ell$ odd. Then $n<\left(k \ell^{\prime} D_{1} / \ell \theta\right)^{\ell /\left(\ell-\ell^{\prime}\right)}$ where $\ell^{\prime}$ is given by Table 3 below. (For example, by Table 3, we understand that if $\ell=7$, then $\ell^{\prime}=5$ for $4 \leqslant k \leqslant 8$ and $\ell^{\prime}=4$ for $k \geqslant 9$.)

Proof. Suppose that (1.1) holds with $n \geqslant\left(k \ell^{\prime} D_{1} / \ell \theta\right)^{\ell /\left(\ell-\ell^{\prime}\right)}$ where $\ell^{\prime}$ is given in Table 3. Then (6.3) and the hypothesis of Lemma 11 are valid. Therefore, we derive from Lemmas 11 and 10 that $k<11380, a_{i}$ for $0 \leqslant i<k$ are distinct and (6.4) is valid. Now we proceed as in the proof of [SS01, Lemma 8]. We illustrate the proof with an example. Let $\ell=5$. Then $\ell^{\prime}=4$ if $4 \leqslant k \leqslant 8$ and $\ell^{\prime}=3$ if $k \geqslant 9$ by Table 3 . Let $k=4$. Then, $H(d, 4,2,0) \geqslant 4$ by Table 1 . Hence (6.4) is not valid. Thus $k \neq 4$. Suppose that $k=2958$. Then by Table $2, m_{1}=4, m_{2}=12$ and $H(d, 2958,4,12) \geqslant 271$. 
PERFECT POWERS IN ARITHMETIC PROGRESSION

TABLE 3 .

\begin{tabular}{rccccc}
\hline \multicolumn{1}{c}{4} & $4 \leqslant k \leqslant 8$ & $k \geqslant 9$ & $\ell$ & $4 \leqslant k \leqslant 8$ & $k \geqslant 9$ \\
\hline 3 & 2 & 2 & 19 & 11 & 8 \\
5 & 4 & 3 & 21 & 12 & 8 \\
7 & 5 & 4 & 23 & 13 & 9 \\
9 & 6 & 5 & 25 & 14 & 9 \\
11 & 8 & 5 & 27 & 15 & 10 \\
13 & 9 & 6 & 29 & 16 & 10 \\
15 & 10 & 7 & $\geqslant 31$ & $(\ell+1) / 2$ & $(\ell+1) / 2$ \\
17 & 11 & 7 & - & - & - \\
\hline
\end{tabular}

TABLE 4.

\begin{tabular}{lrlr}
\hline \multicolumn{1}{c}{$\ell$} & $k^{*}$ & \multicolumn{1}{c}{$\ell$} & \multicolumn{1}{c}{$k^{*}$} \\
\hline 3 & 331 & 12 & 103 \\
$4,6,15,16$ & 23 & 17,20 & 11 \\
5,7 & 317 & 18 & 13 \\
$8,13,14$ & 47 & $19,21-25,27$ & 7 \\
9 & 53 & $26,28,29$ & 5 \\
10 & 19 & $30-37$ & 4 \\
11 & 113 & - & - \\
\hline
\end{tabular}

Hence (6.4) is not valid. Thus $k \neq 2958$. We exclude all values of $k<11380$ as above. The argument for excluding other values of $\ell \leqslant 29$ is similar. Let $\ell \geqslant 31$. Then $\ell^{\prime}=(\ell+1) / 2$ by Table 3 . Let $Y=H_{1}\left(m_{1}\right)$ or $H_{2}\left(m_{1}, m_{2}\right)$. As above we need to show that (6.4) does not hold for $k<11380$ and $\ell \geqslant 31$. We observe that if (6.4) does not hold for some odd $\ell=\ell_{0}$, then it does not hold for $\ell=\ell_{0}+2$ provided that

$$
Y>\left(1+\frac{2}{\ell_{0}}\right)^{m_{1}}\left(m_{2}+1\right)+\left(\frac{\ell_{0}+1}{2}\right)\left(\left(1+\frac{2}{\ell_{0}}\right)^{m_{1}}-1\right) .
$$

We observe that the right-hand side of (6.8) is a decreasing function of $\ell_{0}$. Hence, it is enough to check that $(6.8)$ is valid and (6.4) is not valid at $\ell_{0}=31$. We carry out this by checking at $\ell_{0}=31$ for all $k<11380$ and for $m_{1}, m_{2}, Y$ as in Tables 1 and 2 .

\section{Variables in (1.1) are bounded}

Let $d \leqslant d_{1}$. We first bound $\ell$ and $k$. To do this, we compare the upper bound for $n+(k-1) d$ obtained in $\S \S 5$ and 6 and the lower bound for $n+(k-1) d$ which can be obtained by using the iterative procedure in $\S 3$. See Lemma 13. Let $\ell$ and $k$ be given. Then we bound $n$ by Lemmas 6 or 12 according to whether $\ell$ is even or odd, respectively. We observe that when $n, d, k, \ell$ are bounded, then $b$ and $y$ are also bounded by (1.1).

Lemma 13. Assume (1.1) with Hypothesis A. Then $\ell \leqslant 37$ and $k \leqslant k^{*}$ with $k^{*}$ given by Table 4 .

Proof. Assume (1.1) with Hypothesis A. Then all $A_{j}$ are distinct by Corollary 1 . Thus Lemma 3 is valid. Suppose that $\ell$ is even and $\ell \geqslant 38$. Then $d \leqslant k^{\ell-1} / 2$. Hence in Lemma 3, we may take $\eta_{i}=\eta_{i}^{\prime}=0$ for every $i$. Furthermore, by (1.3) and Lemma 6 , we get $\delta k^{\ell+1}=n+(k-1) d<$ $k^{2} d^{2} /\left(4 h_{0}\right)+k d$. Thus

$$
\delta<\frac{d^{2}}{4 h_{0} k^{\ell-1}}+\frac{d}{k^{\ell}}
$$




\section{N. Saradha And T. N. Shorey}

We observe that the right-hand side of (7.1) decreases as $k$ or $\ell$ increases. Thus we evaluate the right-hand side at $k=4, \ell=38$ and $d=10^{15}$ to get $\delta<1.4 \times 10^{7}$. On the other hand, we see from Lemma 3 and a remark after the proof of (3.3) that $\delta>4 \times 10^{8}$. Thus $\ell \leqslant 36$.

Let $\ell \leqslant 36$ be given. Suppose that $k>k^{*}$ where $k^{*}$ is given in Table 4 . We check that $d \leqslant k^{\ell-1} / 2$ implying that $\eta_{i}=\eta_{i}^{\prime}=0$ for every $i$. Now we compute the lower bound of $\delta$ by Lemma 3 and the upper bound by (7.1) to see that they are inconsistent. Thus $k \leqslant k^{*}$.

Suppose that $\ell$ is odd and $\ell \geqslant 39$. Then $d \leqslant k^{\ell-1} / 2$ and hence every $\eta_{i}=\eta_{i}^{\prime}=0$ in Lemma 3 . Furthermore, by (1.3) and Lemma 12, we get

$$
\delta k^{\ell+1}=n+(k-1) d<\left(\frac{k \ell^{\prime} D_{1}}{\ell \theta}\right)^{\ell /\left(\ell-\ell^{\prime}\right)}+k d
$$

where $\ell^{\prime}=(\ell+1) / 2$. By the definition of $\theta$ given by $(6.2)$, we find that

$$
\frac{D_{1}}{\theta}=D_{1} \prod_{i=1}^{r} \ell_{i}^{\min \left(e_{i}, \operatorname{ord}_{\ell_{i}}\left(D_{2}\right)\right)} \leqslant D_{1} D_{2}=d .
$$

Thus, by (7.2) and (7.3), we get

$$
\delta<\left(\frac{\ell^{\prime} d}{\ell}\right)^{\ell /\left(\ell-\ell^{\prime}\right)} k^{-\ell+\ell^{\prime} /\left(\ell-\ell^{\prime}\right)}+\frac{d}{k^{\ell}} .
$$

Replacing $\ell^{\prime}$ by $(\ell+1) / 2$ in $(7.4)$, we get

$$
\delta<\left(\frac{(\ell+1) d}{2 \ell}\right)^{2 \ell /(\ell-1)} k^{\left(-\ell^{2}+2 \ell+1\right) /(\ell-1)}+\frac{d}{k^{\ell}} .
$$

We observe that the right-hand side of (7.5) decreases as $k$ or $\ell$ increases. Hence, we evaluate the right-hand side of $(7.5)$ at $\ell=39, k=4$ and $d=10^{15}$ to find that $\delta<2.3 \times 10^{7}$. On the other hand, we get $\delta \geqslant 7 \times 10^{8}$ for $k \geqslant 4$ by Lemma 3 . This is a contradiction. Thus $\ell \leqslant 37$. Let $\ell \leqslant 37$ be given. Suppose that $k>k^{*}$ where $k^{*}$ is given in Table 4 . We check that $d \leqslant k^{\ell-1} / 2$ implying that $\eta_{i}=\eta_{i}^{\prime}=0$ for every $i$. Now we compute the lower bound for $\delta$ by Lemma 3 . Next we turn to an upper bound for $\delta$. For this, we use (7.4) with $\ell^{\prime}$ given by Table 3 when $5 \leqslant \ell \leqslant 29$ and we use (7.2) if $\ell=3$. We observe that (7.5) holds when $31 \leqslant \ell \leqslant 37$ since $\ell^{\prime}=(\ell+1) / 2$. Finally, we check that the lower bound and the upper bound for $\delta$ obtained above are inconsistent.

\section{Algorithm for solving (1.1) when $d$ is large}

In this section, we present an algorithm for finding the solutions of (1.1) whenever $k, \ell, d^{\prime}>0$, $\delta_{1}>0, \delta_{2}>0$ are given such that $1<d \leqslant d^{\prime}$ and $\delta_{1}<\delta<\delta_{2}$. We choose $m_{1}, \alpha_{1}, \ldots, \alpha_{m_{1}}$ suitably and compute $G_{0}$ given by (2.18). We put

$$
\kappa=\left\{\begin{array}{ll}
{\left[\frac{k}{G_{0}-2}\right]} & \text { if } G_{0} \geqslant 3 ; \\
k & \text { otherwise }
\end{array} \text { and } a=p_{1}^{\alpha_{1}-1} \cdots p_{m_{1}}^{\alpha_{m_{1}-1}} .\right.
$$

Below, we give the various steps of the algorithm.

\section{Algorithm 1.}

Step 1. We form the set $W_{1}$ of divisors of $a$.

Step 2. We form the set $W_{2}$ of pairs $\left(A Z_{1}^{\ell}, B Z_{2}^{\ell}\right)$ such that $A, B \in W_{1}$ with $A<B, \operatorname{gcd}(A, B)=1$, $Z_{2} / Z_{1}$ is a convergent in the continued fraction expansion of $(A / B)^{1 / \ell}, Z_{1}, Z_{2}$ do not exceed $\delta_{2}^{1 / \ell} k^{1+1 / \ell}$, least prime factor of $Z_{1} Z_{2}>k$ if $G_{0} \geqslant 3$ and $P\left(Z_{1} Z_{2}\right)>k$ if $G_{0}<3$ and $\left(\left(\delta_{1} k^{\ell+1}-k d^{\prime}\right) / a\right)^{1 / \ell}<Z_{1}$. 


\section{PERFECT POWERS IN ARITHMETIC PROGRESSION}

Step 3. Let

$$
\delta_{1} k^{\ell}-d^{\prime} \leqslant\left(2 \kappa d^{\prime}\right)^{\ell /(\ell-2)} .
$$

Then we carry out $(\mathrm{A})-(\mathrm{C})$ as given below.

(A) We form the set $W_{3}$ of all positive integers that are $\ell$ th powers having all prime factors greater than $k$ and not exceeding $\delta_{4}$, where $\delta_{4} \leqslant \min \left(\left(3 \kappa d^{\prime}\right)^{\ell /(\ell-2)}, \delta_{2} k^{\ell+1}\right)$. We observe that $W_{3}$ contains 1 . We take $W_{4}=W_{3}-\{1\}$ if $G_{0} \geqslant 3$ and $W_{4}=W_{3}$ otherwise.

(B) Let $W_{5}$ be the set of integers of the form $A Z^{\ell}$ with $A \in W_{1}, Z^{\ell} \in W_{4}$ such that $\delta_{1} k^{\ell}-d^{\prime} \leqslant A Z^{\ell} \leqslant \delta_{4}$. Let the elements of $W_{5}$ be arranged in increasing order. Let $W_{5}(i)$ denote the $i$ th element of $W_{5}$.

(C) Let $W_{6}$ be the set of pairs $\left(W_{5}(j), W_{5}(i)\right)$ with $\operatorname{gcd}\left(W_{5}(j), W_{5}(i)\right)=1, P\left(W_{5}(j)\right.$ $\left.W_{5}(i)\right)>k$ if $G_{0}<3$ and $W_{5}(i)-W_{5}(j)<\kappa d^{\prime}$ for $1 \leqslant j<i \leqslant\left|W_{5}\right|$.

Step 4. We form $W_{7}=W_{2}$ if (8.2) does not hold and $W_{7}=W_{2} \cup W_{6}$ otherwise.

Step 5. Let $W_{8}$ be the set of pairs $\left(N_{1}, N_{2}\right) \in W_{7}$ for which the triple $\left(N_{1}, N_{2}, 1\right)$ has Property $P_{2}$. Let $W_{9}$ be the set of pairs $\left(N_{1}, N_{2}\right) \in W_{8}$ for which the triple $\left(N_{1}, N_{2}, r\right)$ has Property $P_{1}$ for some integer $r \geqslant 1$ dividing $N_{2}-N_{1}$.

Now we show that under suitable conditions $W_{9} \neq \emptyset$ whenever (1.1) holds.

Lemma 14. Suppose that (1.1) with Hypothesis A holds. Let $k \geqslant 4, G_{0} \geqslant 2$ and $\delta_{1} k^{\ell} \geqslant a$. Then $W_{9} \neq \emptyset$ where $W_{9}$ is constructed as in the algorithm with $d^{\prime}=d_{1}$.

Proof. Assume (1.1) with Hypothesis A. By Corollary 1, all the $A_{i}$ are distinct. Therefore, there are at least $G_{0}$ of the $A_{i}$ in $W_{1}$, which are composed of $p_{1}, \ldots, p_{m_{1}}$ to the orders not exceeding $\alpha_{1}-1, \ldots, \alpha_{m_{1}}-1$, respectively. Suppose that $G_{0} \geqslant 3$. Then we divide the interval $[0, k)$ into $G_{0}-2$ sub intervals

$$
\left[0, \frac{k}{G_{0}-2}\right), \ldots,\left[\frac{\left(G_{0}-3\right) k}{G_{0}-2}, k\right)
$$

of length $k /\left(G_{0}-2\right)$. We find that there exists a sub interval from (8.3) containing two integers $0<i_{0}<j_{0}<k$ such that $A_{i_{0}}, A_{j_{0}}$ are in $W_{1}$. Since $G_{0} \geqslant 2$ there always exist two terms of $\Delta$, say, $n+i d=A_{i} X_{i}^{\ell}$ and $n+j d=A_{j} X_{j}^{\ell}$ with $j>i$ and $A_{i}, A_{j}$ in $W_{1}$. By (8.1), we find

$$
(j-i) d=A_{j} X_{j}^{\ell}-A_{i} X_{i}^{\ell} \quad \text { with } 0<j-i<\kappa
$$

where $i>0$ whenever $G_{0} \geqslant 3$. Suppose that $X_{j}=1$. Then since $j>0$, we have $a \geqslant A_{j}=A_{j} X_{j}^{\ell}=$ $n+j d>d$. Hence, $a \geqslant n+j d>n>\delta_{1} k^{\ell+1}-(k-1) d>\delta_{1} k^{\ell+1}-(k-1) a$ contradicting our assumption. Thus $X_{j} \neq 1$. Similarly, we see that $X_{i} \neq 1$ whenever $i \neq 0$. Thus we always have $P\left(X_{i} X_{j}\right)>k$ and if $G_{0} \geqslant 3$, then the least prime factor of $X_{i} X_{j}>k$. We note that $A_{i}, A_{j}$ are coprime to $d$. Hence by (8.4), we get

$$
\alpha=\operatorname{gcd}\left(A_{i}, A_{j}\right)<k .
$$

Furthermore, we put $A_{i}^{\prime}=\alpha^{-1} A_{i}, A_{j}^{\prime}=\alpha^{-1} A_{j}$. By dividing both sides of (8.4) by $\operatorname{gcd}\left(A_{i}, A_{j}\right)$, we get

$$
A_{\mu}^{\prime} X_{\mu}^{\ell}-A_{\nu}^{\prime} X_{\nu}^{\ell}= \pm r d
$$

where $0<r<\kappa / \alpha,(\mu, \nu)=(i, j)$ or $(j, i), A_{\mu}^{\prime}>A_{\nu}^{\prime}$ are in $W_{1}$ and $\operatorname{gcd}\left(A_{\mu}^{\prime}, A_{\nu}^{\prime}\right)=1$. Furthermore, by (8.5), we see that

$$
\delta_{1} k^{\ell}-d_{1}<A_{\mu}^{\prime} X_{\mu}^{\ell}, A_{\nu}^{\prime} X_{\nu}^{\ell}<\delta_{2} k^{\ell+1}
$$




\section{N. Saradha And T. N. Shorey}

Therefore $X_{\mu}, X_{\nu}$ do not exceed $\delta_{2}^{1 / \ell} k^{1+1 / \ell}$ and they are bounded from below by $\left(\left(\delta_{1} k^{\ell+1}-\right.\right.$ $\left.\left.k d_{1}\right) / a\right)^{1 / \ell}$. Suppose that $(8.2)$ does not hold. Then we find by $(8.7)$ that

$$
A_{\nu}^{\prime} X_{\nu}^{\ell-2} \geqslant\left(A_{\nu}^{\prime} X_{\nu}^{\ell}\right)^{(\ell-2) / \ell}>\left(\delta_{1} k^{\ell}-d_{1}\right)^{(\ell-2) / \ell}>2 \kappa d_{1} .
$$

From (8.6) we get

$$
\left|\frac{A_{\nu}^{\prime}}{A_{\mu}^{\prime}}-\frac{X_{\mu}^{\ell}}{X_{\nu}^{\ell}}\right|<\frac{\kappa d_{1}}{A_{\mu}^{\prime} X_{\nu}^{\ell}}
$$

which, by (8.8), implies that

$$
\left|\left(\frac{A_{\nu}^{\prime}}{A_{\mu}^{\prime}}\right)^{1 / \ell}-\frac{X_{\mu}}{X_{\nu}}\right|<\frac{\kappa d_{1}}{A_{\mu}^{\prime} X_{\nu}^{\ell}}\left(\frac{A_{\mu}^{\prime}}{A_{\nu}^{\prime}}\right)^{1-1 / \ell}<\frac{\kappa d_{1}}{A_{\nu}^{\prime} X_{\nu}^{\ell}}<\frac{1}{2 X_{\nu}^{2}} .
$$

Thus $X_{\mu} / X_{\nu}$ is a convergent in the continued fraction expansion of $\left(A_{\nu}^{\prime} / A_{\mu}^{\prime}\right)^{1 / \ell}$, see [NZ80, p. 161]. Hence $\left(A_{\nu}^{\prime} X_{\nu}^{\ell}, A_{\mu}^{\prime} X_{\mu}^{\ell}\right) \in W_{2}=W_{7}$. We observe that $\alpha A_{\nu}^{\prime} X_{\nu}^{\ell}, \alpha A_{\mu}^{\prime} X_{\mu}^{\ell}$ are two terms of $\Delta$ and hence the triple $\left(\alpha A_{\nu}^{\prime} X_{\nu}^{\ell}, \alpha A_{\mu}^{\prime} X_{\mu}^{\ell}, \alpha r\right)$ has Property $P_{1}$. Since $\alpha<k$, we conclude that the triple $\left(A_{\nu}^{\prime} X_{\nu}^{\ell}, A_{\mu}^{\prime} X_{\mu}^{\ell}, r\right)$ has Property $P_{1}$. Furthermore, we observe that $\left(A_{\nu}^{\prime} X_{\nu}^{\ell}, A_{\mu}^{\prime} X_{\mu}^{\ell}, 1\right)$ also has Property $P_{2}$. Thus the pair $\left(A_{\nu}^{\prime} X_{\nu}^{\ell}, A_{\mu}^{\prime} X_{\mu}^{\ell}\right) \in W_{9}$, which proves the assertion.

Thus we may suppose that (8.2) holds. Furthermore, if $A_{\nu}^{\prime} X_{\nu}^{\ell-2}>2 \kappa d_{1}$, then we argue as in the previous paragraph to see that $\left(A_{\nu}^{\prime} X_{\nu}^{\ell}, A_{\mu}^{\prime} X_{\mu}^{\ell}\right) \in W_{9}$ yielding the assertion. Thus, we may suppose that $A_{\nu}^{\prime} X_{\nu}^{\ell-2} \leqslant 2 \kappa d_{1}$. Hence

$$
A_{\nu}^{\prime} X_{\nu}^{\ell} \leqslant\left(A_{\nu}^{\prime} X_{\nu}^{\ell-2}\right)^{\ell /(\ell-2)} \leqslant\left(2 \kappa d_{1}\right)^{\ell /(\ell-2)},
$$

which, together with (8.7), implies that $A_{\nu}^{\prime} X_{\nu}^{\ell} \in W_{5}$. Let $A_{\nu}^{\prime} X_{\nu}^{\ell}=W_{5}(i)$ for some $i \geqslant 1$. We see from (8.9) and (8.6) that $A_{\mu}^{\prime} X_{\mu}^{\ell} \leqslant A_{\nu}^{\prime} X_{\nu}^{\ell}+\kappa d_{1} \leqslant\left(3 \kappa d_{1}\right)^{\ell /(\ell-2)}$ and hence $A_{\mu}^{\prime} X_{\mu}^{\ell} \in W_{5}$. Thus by (8.6), the pair $\left(A_{\nu}^{\prime} X_{\nu}^{\ell}, A_{\mu}^{\prime} X_{\mu}^{\ell}\right) \in W_{6}$. Arguing as earlier, we see that $\left(A_{\nu}^{\prime} X_{\nu}^{\ell}, A_{\mu}^{\prime} X_{\mu}^{\ell}\right) \in W_{9}$ which proves the assertion.

\section{Proof of Theorem 1 when $\ell>3$}

We assume (1.1) with Hypothesis $\mathrm{A}$ and $\ell>3$. Then $k \geqslant 4$. By Lemma 13 , we see that $\ell \leqslant 37$ and $k \leqslant k^{*}$ with $k^{*}$ given by Table 4 . We apply Algorithm 1 with $d^{\prime}=d_{1}$ to exclude all of these values of $\ell$ and $k$ as follows. For all values of $\ell$, we choose $m_{1}, \alpha_{1}, \ldots, \alpha_{m_{1}}$, as below. We give the choices for $k \leqslant k^{*}, k$ prime or $k=4$.

$$
\begin{aligned}
& k=4: m_{1}=2, \alpha_{1}=\alpha_{2}=2 ; \\
& 5 \leqslant k \leqslant 10: m_{1}=2, \quad \alpha_{1}=3, \quad \alpha_{2}=2 ; \\
& 11 \leqslant k \leqslant 28: m_{1}=3, \quad \alpha_{1}=4, \quad \alpha_{2}=\alpha_{3}=2 ; \\
& 29 \leqslant k \leqslant 36: m_{1}=3, \quad \alpha_{1}=4, \quad \alpha_{2}=3, \quad \alpha_{3}=2 ; \\
& 37 \leqslant k \leqslant 66: m_{1}=3, \quad \alpha_{1}=5, \quad \alpha_{2}=3, \quad \alpha_{3}=2 ; \\
& 67 \leqslant k \leqslant 100: m_{1}=4, \quad \alpha_{1}=4, \quad \alpha_{2}=3, \quad \alpha_{3}=\alpha_{4}=2 ; \\
& 101 \leqslant k \leqslant 198: m_{1}=5, \quad \alpha_{1}=4, \quad \alpha_{2}=3, \quad \alpha_{3}=\alpha_{4}=\alpha_{5}=2 ; \\
& 199 \leqslant k \leqslant 270: m_{1}=5, \quad \alpha_{1}=5, \quad \alpha_{2}=3, \quad \alpha_{3}=\alpha_{4}=\alpha_{5}=2 ; \\
& 271 \leqslant k \leqslant 331: m_{1}=5, \quad \alpha_{1}=5, \quad \alpha_{2}=\alpha_{3}=3, \quad \alpha_{4}=\alpha_{5}=2 .
\end{aligned}
$$




\section{PERFECT POWERS IN ARITHMETIC PROGRESSION}

By these choices of $m_{1}, \alpha_{1}, \ldots, \alpha_{m_{1}}$, we find that $G_{0} \geqslant 4$ except for $k=4,5,7,19,23$ in which cases $G_{0}=2,2,2,3,3$, respectively. By (2.7) and $\ell \geqslant 4$, we see that $\delta>\delta_{1}$ where $\delta_{1} k^{\ell} \geqslant p_{\pi(k)+\chi_{0}}^{4} / k \geqslant a$ with $a$ given by (8.1). Thus the conditions of Lemma 14 are satisfied. We now explain the rest of the algorithm by means of an example.

We take $\ell=5$. Then $d_{1}=5 \cdot 10^{4}$ and $k^{*}=317$. For every $k \leqslant k^{*}$, we compute $G_{0}$ and by Lemma 3 , we find $\delta_{1}$. We observe that (8.2) is valid only for $k \leqslant 61$. Thus

$$
W_{7}= \begin{cases}W_{2} \cup W_{6} & \text { for } 4 \leqslant k \leqslant 61 \\ W_{2} & \text { for } 67 \leqslant k \leqslant 317 .\end{cases}
$$

We fix $k=11$. Then $m_{1}=3, \alpha_{1}=4, \alpha_{2}=\alpha_{3}=2$ and $G_{0}=4$. Thus $\kappa=5, a=2^{3} \cdot 3 \cdot 5$. We apply Lemma 3 to get $\delta>7=\delta_{1}$. By Lemma 12, we find that

$$
\frac{n+(k-1) d}{k^{6}} \leqslant\left(\left(33 \cdot 10^{4}\right)^{5 / 2}+5 \cdot 10^{5}\right) / 11^{6}<35312523=\delta_{2} .
$$

By (9.1), we need to form the sets $W_{2}$ as well as $W_{6}$. As in Step 1 , we first form the set $W_{1}$ of divisors of $2^{3} \cdot 3 \cdot 5$. Then we form the set $W_{2}$. For this, we fix $(A, B)$ with

$$
A, B \in W_{1}, A<B, \operatorname{gcd}(A, B)=1 .
$$

Next we find all integers $Z_{1}, Z_{2}$ such that $Z_{1}, Z_{2} \leqslant \delta_{2}^{1 / 5} 11^{6 / 5} \leqslant 575, Z_{1}>\left(\left(7 \cdot 11^{6}-5 \cdot 10^{5}\right) /\right.$ $\left.2^{3} \cdot 3 \cdot 5\right)^{1 / 5}>9$, the least prime factor of $Z_{1} Z_{2}>11$ and $Z_{2} / Z_{1}$ is a convergent in the continued fraction expansion of $(A / B)^{1 / 5}$. Then we form the set $W_{2}(A, B)$ of all pairs $\left(A Z_{1}^{5}, B Z_{2}^{5}\right)$. Finally we put $W_{2}=\bigcup W_{2}(A, B)$ where the union is taken over all the pairs $(A, B)$ satisfying $(9.2)$. We get

$$
W_{2}=\left\{\left(47^{5}, 2^{3} \cdot 31^{5}\right),\left(3 \cdot 19^{5}, 2^{2} \cdot 5 \cdot 13^{5}\right)\right\}
$$

We proceed to carry out Step $3(\mathrm{~A})-(\mathrm{C})$. We have $\delta_{4}=6.2 \times 10^{9}$. Thus $W_{3}$ is the set of integers of the form $Z^{5}$ with the least prime factor of $Z$ exceeding 11 and $Z \leqslant 90$. Hence, $W_{4}$ is the set of fifth powers of all primes greater than 11 and less than or equal to 89 . Then $W_{5}$ is the set of integers of the form $A Z^{5}$ with $A \in W_{1}, Z^{5} \in W_{4}$ such that $1077357 \leqslant \delta_{1} k^{\ell}-d_{1} \leqslant A Z^{\ell} \leqslant 6.2 \times 10^{9}$. Now we find all pairs $\left(W_{5}(j), W_{5}(i)\right)$ with $\operatorname{gcd}\left(W_{5}(j), W_{5}(i)\right)=1$ and $W_{5}(i)-W_{5}(j) \leqslant \kappa d_{1}=25 \cdot 10^{4}$ for $1 \leqslant j<i \leqslant\left|W_{5}\right|$. We get

$$
W_{6}=\left\{\left(2^{2} \cdot 13^{5}, 17^{5}\right),\left(19^{5}, 2 \cdot 3 \cdot 13^{5}\right),\left(2^{2} \cdot 17^{5}, 3 \cdot 5 \cdot 13^{5}\right),\left(3 \cdot 19^{5}, 2^{2} \cdot 5 \cdot 13^{5}\right),\left(31^{5}, 2^{2} \cdot 5 \cdot 17^{5}\right)\right\} .
$$

Then $W_{7}$ is the union of the sets in (9.3) and (9.4). Among the pairs $\left(N_{1}, N_{2}\right)$ in $W_{7}$, we find that Property $P_{2}$ holds for $\left(N_{1}, N_{2}, 1\right)$ only when $\left(N_{1}, N_{2}\right)$ is in the set

$$
W_{8}=\left\{\left(19^{5}, 2 \cdot 3 \cdot 13^{5}\right),\left(2^{2} \cdot 17^{5}, 3 \cdot 5 \cdot 13^{5}\right),\left(31^{5}, 2^{2} \cdot 5 \cdot 17^{5}\right)\right\} .
$$

For each pair $\left(N_{1}, N_{2}\right)$ in $W_{8}$, we check that the triple $\left(N_{1}, N_{2}, r\right)$ does not have Property $P_{1}$ for any integer $r \geqslant 1$ dividing $N_{2}-N_{1}$. Thus $W_{9}=\emptyset$, which is not possible by Lemma 14 . Thus $k \neq 11$. All other values of $k$ with $4 \leqslant k \leqslant 317$ are excluded similarly. Finally we exclude all other values of $\ell$ as above.

\section{Algorithm for solving (1.1) when $d$ is small}

We give an algorithm for finding solutions of (1.1) when $d$ is small and this is more efficient than Algorithm 1. This algorithm is a modified extension of the algorithm given in [SS03]. Let $k, \ell$, $d^{\prime}>0, \delta_{1}>0, \delta_{2}>0$ be given such that $1<d \leqslant d^{\prime}$ and $\delta_{1}<\delta<\delta_{2}$. Let $d$ be fixed. We now give the various steps of the algorithm. 


\section{N. Saradha And T. N. Shorey}

Algorithm 2.

Step $\mathbf{1}^{\prime}$. We have $n+(k-1) d>\delta_{1} k^{\ell+1}$. We use (3.1) if $d \geqslant \delta_{1} k^{\ell} / 2$ and, otherwise, we use (3.2) to estimate $|I| \geqslant \alpha^{\prime}$, say. Let $\chi_{1}=\max \left(\alpha^{\prime}, 2\right)$ and $p_{\pi(k)+\chi_{1}}=Q_{0}$. For any integer $i>0$, we put

$$
\begin{aligned}
& s_{1}(i)=\left[\frac{\delta_{1} k^{\ell+1}-k d}{i^{\ell}}\right]+1 ; \quad s_{2}(i)=\left[\frac{\delta_{2} k^{\ell+1}}{i^{\ell}}\right] ; \\
& s_{3}(i)=\left[\frac{Q_{0}^{\ell} k^{\ell}}{i^{\ell}}\right]+1 ; \quad s_{4}(i)=\min \left(s_{2}(i), s_{3}(i)\right) .
\end{aligned}
$$

Now we find all primes $Q$ such that

$$
Q_{0} \leqslant Q \leqslant \min \left(Q_{0} k, \delta_{2}^{1 / \ell} k^{1+1 / \ell}\right) .
$$

For each $Q$ in (10.1) we form the set

$$
D_{Q}^{\prime}=\left\{t Q^{l} \mid \operatorname{gcd}\left(t Q^{l}, d\right)=1, P(t) \leqslant k, s_{1}(Q) \leqslant t \leqslant s_{4}(Q)\right\} .
$$

We put $E^{\prime}=\bigcup D_{Q}^{\prime}$ where the union is taken over all $Q$ satisfying (10.1). Furthermore, if $Q_{0}<\left(\delta_{2} k\right)^{1 / \ell}$, then we find all primes $Q$ such that

$$
Q_{0} \leqslant Q \leqslant \delta_{2}^{1 / \ell} k^{1+1 / \ell}
$$

For each $Q$ in (10.2) we form the set

$$
D_{Q}^{\prime \prime}=\left\{t Q^{l} \mid \operatorname{gcd}\left(t Q^{l}, d\right)=1, s_{3}(Q) \leqslant t \leqslant s_{2}(Q) \text { and } t Q^{l} \text { has Property } P_{0}\right\} .
$$

We put $E^{\prime \prime}=\bigcup D_{Q}^{\prime \prime}$ where the union is taken over all $Q$ satisfying (10.2). Finally we put

$$
E_{d}=E^{\prime} \cup E^{\prime \prime} \quad \text { if } Q_{0}<\left(\delta_{2} k\right)^{1 / \ell} ; \quad E=E^{\prime} \quad \text { otherwise. }
$$

Step 2'. Let $E_{d, 0}=E_{d}$. We take $E_{d, 1}^{\prime}$ to be the set of $N \in E_{d, 0}$ for which $N+d$ as well as $N-d$ do not have Property $P_{0}$. We put $E_{d, 1}=E_{d, 0}-E_{d, 1}^{\prime}$. Next, we construct $E_{d, 2}^{\prime}$ to be the set of $N \in E_{d, 1}$ for which $N+2 d$ does not have Property $P_{0}$ and at least one of $N-d$, $N-2 d$ does not have Property $P_{0}$. We put $E_{d, 2}=E_{d, 1}-E_{d, 2}^{\prime}$. We proceed inductively to construct the sets $E_{d, 3}^{\prime}, E_{d, 3}, \ldots$ We observe that

$$
E_{d, 0} \supseteq E_{d, 1} \supseteq E_{d, 2} \supseteq \cdots \text {. }
$$

Thus, $N \in E_{d, i}$ implies that $N \notin E_{d, i}^{\prime}$ which means that either $N+i d$ has Property $P_{0}$ or every one of $N-d, N-2 d, \ldots, N-i d$ has Property $P_{0}$.

Step $3^{\prime}$. We construct the sequence (10.3) for every $d \leqslant d^{\prime}$.

Lemma 15. Assume (1.1) with Hypothesis A. Let $k \geqslant 4$ and $\delta_{1}<\delta<\delta_{2}$. Suppose that $d \leqslant d_{1}$ is fixed. Then $E_{d, i} \neq \emptyset$ for $1 \leqslant i \leqslant\left[\frac{k}{2}\right]$.

Proof. Assume (1.1) with Hypothesis A and let $\delta_{1}<\delta<\delta_{2}$. Then we see that there is a term in $\Delta$, say $n+h d, 0 \leqslant h<k$ which is divisible by a prime $Q_{1} \geqslant p_{\pi(k)+\chi_{1}}=Q_{0}$ to an $\ell$ th power. Thus $n+h d=t_{1} Q_{1}^{\ell}$ where $t_{1}$ is a positive integer. Furthermore, $\operatorname{gcd}\left(t_{1} Q_{1}^{l}, d\right)=1 \operatorname{since} \operatorname{gcd}(n, d)=1$. Suppose that $Q_{0} \geqslant\left(\delta_{2} k\right)^{1 / \ell}$. Then $t_{1} \delta_{2} k \leqslant t_{1} Q_{0}^{\ell} \leqslant t_{1} Q_{1}^{\ell}<\delta_{2} k^{\ell+1}$ implying $t_{1}<k^{\ell}$. Since $t_{1} Q_{1}^{\ell}$ is a term of $\Delta$, it has Property $P_{0}$. Hence $P\left(t_{1}\right) \leqslant k$. Furthermore, since $\delta_{1}<\delta<\delta_{2}$ and $Q_{0}^{\ell} k^{\ell} \geqslant \delta_{2} k^{\ell+1}$, we have $s_{1}\left(Q_{1}\right) \leqslant t_{1} \leqslant\left[\delta_{2} k^{\ell+1} / Q_{1}^{\ell}\right]=s_{4}\left(Q_{1}\right)$. Also $Q_{1}$ satisfies (10.1). Thus $t_{1} Q_{1}^{\ell} \in E^{\prime}$. Suppose that $Q_{0}<\left(\delta_{2} k\right)^{1 / \ell}$. If $t_{1} Q_{1}^{\ell}$ satisfies $Q_{0}^{\ell} k^{\ell}<t_{1} Q_{1}^{\ell}<\delta_{2} k^{\ell+1}$, then we see that $t_{1} Q_{1}^{\ell} \in E^{\prime \prime}$. If $t_{1} Q_{1}^{\ell} \leqslant Q_{0}^{\ell} k^{\ell}$, then $t_{1} Q_{1}^{\ell} \in E^{\prime}$. Hence $n+h d \in E_{d, 0}$. By (1.1), we see that $n+(h+j) d$ has Property $P_{0}$ for $0 \leqslant j<k-h$. Also $n+(h-j) d$ has Property $P_{0}$ for $0 \leqslant j \leqslant h$. Let $h \leqslant[k / 2]$. Then $n+h d \in E_{d, i}$ for every $0 \leqslant i \leqslant[k / 2]$ since $k=4$ or $k$ is prime. Suppose that $[k / 2]<h<k$. Then we 


\section{PERFECT POWERS IN ARITHMETIC PROGRESSION}

consider $n+(h-[k / 2]) d=n+h^{\prime} d$ with $0<h^{\prime} \leqslant[k / 2]$. We see that $n+h^{\prime} d$ is a term of $\Delta$ and arguing as earlier we get $n+h^{\prime} d \in E_{d, i}$ for every $0 \leqslant i \leqslant[k / 2]$. This proves the assertion.

\section{Proof of Theorem 1 when $\ell=3$}

We assume (1.1) with Hypothesis $\mathrm{A}$ and $\ell=3$. Let $k \geqslant 4$. Then by Lemma 9 , we have

$$
d \in\{7,13,14,19,21,26,28\} .
$$

Furthermore, by (7.2) and Lemma 3, we see that $k$ is bounded above by $47,131,47,263,331,131,47$ according as $d=7,13,14,19,21,26,28$, respectively. We apply Algorithm 2 to exclude all of these values of $k$ and $d$. We explain by means of examples. Let $d=19$. First we take $k=109$. By Lemma 3 , we see that $\delta>4.8804=\delta_{1}$. By $(7.2)$, we get $\delta<18.65=\delta_{2}$. We compute $\chi_{1}=\alpha^{\prime}=56$. Thus $Q_{0}=439$. We see that $Q_{0}>\left(\delta_{2} k\right)^{1 / \ell}$. Thus we need to compute $E_{d}=E^{\prime}$. First we find all the primes $Q$ between 439 and 1380 . Then we form $D_{Q}^{\prime}$. For instance, when $Q=439$, we get $s_{1}(Q)=9, s_{4}(Q)=31$ and $D_{439}^{\prime}=\left\{t 439^{3} \mid 9 \leqslant t \leqslant 31, t \neq 19\right\}$. We now construct $E_{d}$ and follow Step $2^{\prime}$. We find that $E_{d, 1}=\left\{3^{2} \cdot 479^{3}, 2 \cdot 7 \cdot 449^{3}, 2 \cdot 3 \cdot 751^{3}\right\}$ and $E_{d, 2}=\emptyset$. This contradicts Lemma 15. Thus $k \neq 109$. Next we take $k=4$. Then $\delta>1.339=\delta_{1}$. By $(7.2)$, we get $\delta<509=\delta_{2}$. Furthermore, $\chi_{1}=\alpha^{\prime}=2$. Thus $Q_{0}=7$. We see that $Q_{0}<(4 \times 509)^{1 / 3}$. Thus we need to compute $E_{d}=E^{\prime} \cup E^{\prime \prime}$. For the primes between 7 and 23, we compute $D_{Q}^{\prime}$ as well as $D_{Q}^{\prime \prime}$. For primes between 29 and 47 , we compute $D_{Q}^{\prime \prime}$. For instance, suppose $Q=29$, then we find that $s_{3}(Q)=1, s_{2}(Q)=5$ and $D_{Q}^{\prime \prime}=\left\{t 29^{3} \mid 1 \leqslant t \leqslant 5, t \neq 5\right\}$. We follow Step $2^{\prime}$ to get $E_{d, 1}=\emptyset$. Thus $k \neq 4$. All other values of $k$ are excluded similarly. We exclude all the values of $d$ in (11.1) as above.

Let $k=3$. By the result of Győry [Gyö99], we have $3 \nmid d$. Now we omit the one term in $\Delta$ divisible by 3 . Then we see from (1.1) that

$$
N(N+i d)=b_{1}^{\prime} y_{1}^{3}, \quad i=1,2, \quad b_{1}^{\prime}=1,2,4,
$$

where $N=n$ or $n+d$ if $i=1, N=n$ if $i=2$ and $y_{1}$ is some positive integer. Suppose that $d$ is even. Then $N$ and $N+i d$ are both odd and by (11.2) we have $N=u^{3}, N+i d=v^{3}$ for some positive integers $u$ and $v$. Thus $60 \geqslant i d=v^{3}-u^{3}$ implying that $(u, v)=(1,3)$. Thus $n=1, d=26$, which is not possible by (1.1). Hence, we may assume that $d$ is odd. Thus

$$
d \in\{5,7,11,13,17,19,23,25,29\} .
$$

We put $X_{1}=N+i d / 2$. Then (11.2) becomes $X_{1}^{2}-i^{2} d^{2} / 4=b_{1}^{\prime} y_{1}^{3}$. We see that this equation can be rewritten as $X^{2}=Y^{3}+\left(b_{2}^{\prime} d\right)^{2}$ with $b_{2}^{\prime} \in\{1,2,4\}$. Now we use SIMATH to find all the integral solutions of this elliptic equation when $d$ is given by (11.3). We check that none of the integral solutions yield any solution to (1.1).

\section{REFERENCES}

Dic52 L. E. Dickson, History of the theory of numbers, vol. II (Chelsea, New York, 1952).

DG95 H. Darmon and A. Granville, On the equations $z^{m}=F(x, y)$ and $A x^{p}+B y^{q}=C z^{r}$, Bull. London Math. Soc. 27 (1995), 513-543.

Erd39 P. Erdős, Note on the product of consecutive integers (II), J. London Math. Soc. (2) 14 (1939), 245-249.

ES75 P. Erdős and J. L. Selfridge, The product of consecutive integers is never a power, Illinois J. Math. 19 (1975), 292-301.

FH01 P. Filakovszky and L. Hajdu, The resolution of the Diophantine equation $x(d+d) \cdots(x+(k-1) d)=$ by $y^{2}$ for fixed d, Acta Arith. 98 (2001), 151-154.

Győ98 K. Győry, On the Diophantine equation $n(n+1) \cdots(n+k-1)=b x^{\ell}$, Acta Arith. 83 (1998), 87-92. 


\section{PERFECT POWERS IN ARITHMETIC PROGRESSION}

Győ99 K. Győry, Power values of products of consecutive integers and binomial coefficients, in Number theory and its applications (Kyoto, 1997), Dev. Math., vol. 2 (Kluwer, Dordrecht, 1999), 145-156.

Mar85 R. Marszalek, On the product of consecutive elements of an arithmetic progression, Monatsh. Math. 100 (1985), 215-222.

MS03 A. Mukhopadhyay and T. N. Shorey, Almost squares in arithmetic progression II, Acta Arith. 110 (2003), 1-14.

NZ80 I. Niven and H. S. Zuckerman, An introduction to the theory of numbers, fourth edition (Wiley, New York, 1980).

RS62 B. Rosser and L. Schoenfeld, Approximate formulas for some functions of prime numbers, Illinois J. Math. 6 (1962), 64-94.

Sar97 N. Saradha, On perfect powers in products with terms from arithmetic progressions, Acta Arith. 82 (1997), 147-172.

Sar98 N. Saradha, Squares in products with terms in an arithmetic progression, Acta Arith. 86 (1998), $27-43$.

Sho00 T. N. Shorey, Exponential Diophantine equations involving products of consecutive integers and related equations, in Number Theory, eds R. P. Bambah, V. C. Dumir and R. J. Hans-Gill, Trends Math. (Birkhäuser, Basel, 2000), 463-495.

Sho02a T. N. Shorey, Powers in arithmetic progression, in A panorama in number theory or the view from Baker's garden, ed. G. Wüstholz (Cambridge University Press, Cambridge, 2002), 325-336.

Sho02b T. N. Shorey, Powers in arithmetic progression II, in New aspects of analytic number theory (Kyoto, 2001), Sūrikaisekikenkyūshu Kōkyūroku, vol. 1274 (Kyoto University, Kyoto, 2002).

SS01 N. Saradha and T. N. Shorey, Almost perfect powers in arithmetic progression, Acta Arith. 99 (2001), 363-388.

SS03 N. Saradha and T. N. Shorey, Almost squares in arithmetic progression, Compositio Math. 138 (2003), 73-111.

SST02 N. Saradha, T. N. Shorey and R. Tijdeman, Some extensions and refinements of a theorem of Sylvester, Acta Arith. 102 (2002), 167-181.

ST90 T. N. Shorey and R. Tijdeman, On the greatest prime factor of an arithmetical progression. A tribute to Paul Erdős, eds A. Baker, B. Bollobás and A. Hajnal (Cambridge University Press, Cambridge, 1990), 385-389.

Ste75 C. L. Stewart, The greatest prime factor of $a^{n}-b^{n}$, Acta. Arith. 26 (1975), 427-433.

Tij89 R. Tijdeman, Diophantine equations and Diophantine approximations, in Number Theory and Applications (Banff, 1988) (Kluwer, Dordrecht, 1989), 215-243.

N. Saradha saradha@math.tifr.res.in

School of Mathematics, Tata Institute of Fundamental Research, Homi Bhabha Road, Mumbai 400 005, India

T. N. Shorey shorey@math.tifr.res.in

School of Mathematics, Tata Institute of Fundamental Research, Homi Bhabha Road, Mumbai 400 005, India 